GEOLOGICAL SURVEY

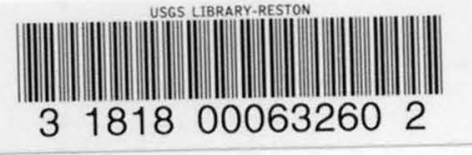

\title{
Analytical results, geology, and sample locality map \\ of mercury-sul fur-gypsum mineralization \\ at Crater, Inyo County, California
}

By

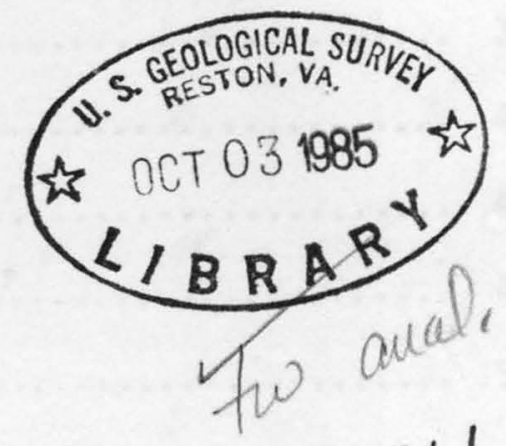

M. S. Erickson, S. P. Marsh, and

T. A. Roemer

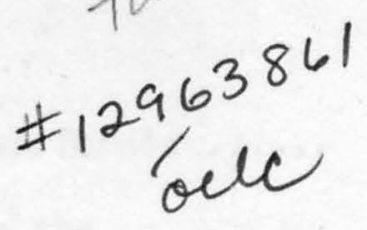

Open-File Report 85-512

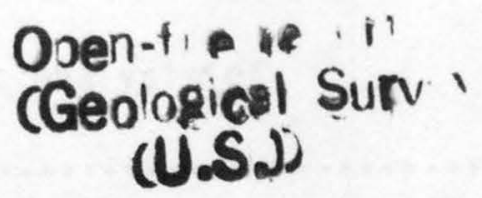

This report is preliminary and has not been reviewed for conformity with U.S. Geological Survey editorial standards and stratigraphic nomenclature. Any use of trade names is for descriptive purposes only and does not imply endorsement by the USGS. 
Introduction.

Methods of study $\ldots \ldots \ldots \ldots \ldots \ldots \ldots \ldots \ldots \ldots \ldots \ldots \ldots \ldots \ldots \ldots \ldots \ldots \ldots$

Sample Media...................................... 1

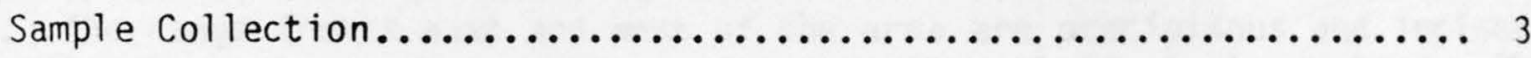

Rock samples.................................. 3

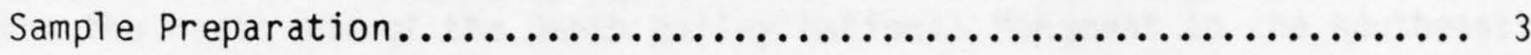

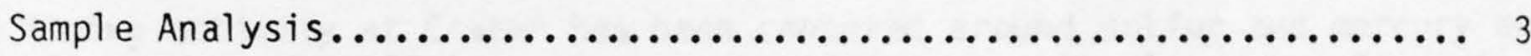

Spectrographic method.............................. 3

Chemical methods.............................. 4

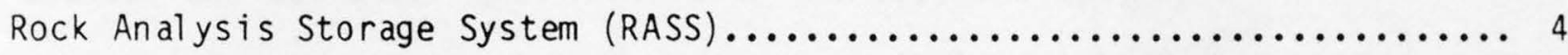

Description of Data Tables.............................. 4

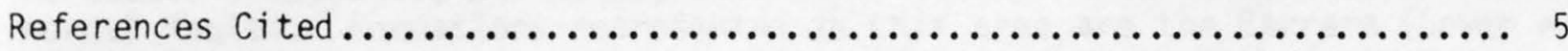

\section{ILLUS TRATIONS}

FIGURE 1. Location map of the Crater Area, Inyo County, California ..... 2 PLATE 1. Map showing geology and rock sample localities in the Crater Mercury-Sulfur-Gypsum Mineral ized Area, Inyo County,

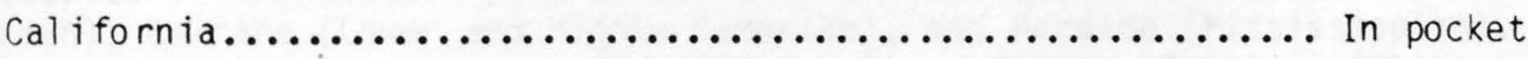

\section{TABLES}

TABLE 1. Limits of determination for spectrographic analysis of

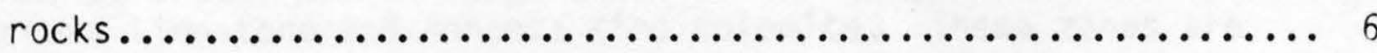

TABLE 2. Chemical methods used.......................... 7

TABLE 3. Descriptions of rock samples..................... 8

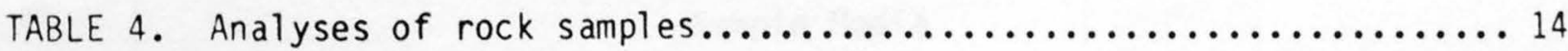




\section{INTRODUCTION}

The Crater mercury-sulfur-gypsum mineralized area is located in eastcentral California al ong the crest of the Last Chance Range, west of the north end of Death Valley (fig. 1). The area is in the northwest quarter of the Last Chance Range 15-minute quadrangle and occupies the area between 11739 and 11745 longitude and 3710 and 3715 latitude. The area studied lies between $5000(1525 \mathrm{~m})$ and $6000(1830 \mathrm{~m})$ feet above sea level. Relief is generally moderate but can be extreme in some places, as at Hanging Rock Canyon (plate 1). The climate is arid, and there are no active streams in the area. The range fronts east and west of the area are precipitous and incised by many steep canyons, whereas the range crest has relatively low relief. The old abandoned town and mine site of Crater lie in this area of low relief. Access to the Crater area is by paved and dirt roads from Big Pine to the west or from the north end of the Death Valley National Monument to the southeast.

Mining activity at Crater has been centered around sulfur and mercury and dates from the early 1900's. At one time, Crater was the largest sulfur mine in Cal ifornia (Wrucke and others, 1984). All that remains now are several 1 arge open pits and some relic equipment. About two miles northeast of Crater is the abandoned El Capitan mercury mine, and numerous prospect pits, small shafts, and bulldozer cuts occur over the entire area.

The geology of the Crater area can be divided into two major terranes. The eastern part of the area consists of a section of relatively flat-lying Paleozoic limestones, dolomites, and shales cut by north-to-northwest-trending normal faults. Formations represented in this area are the Carrara (Lower and Middle Cambrian), Bonanza King Dolomite (Middle and Upper Cambrian), Nopah (Upper Cambrian and Lower Ordovician), and Rest Spring Shale (Mississippian). The other geologic terrane occupies the western part of the area and is separated from the eastern terrane by a north-south trending normal fault. The western terrane has undergone extreme structural deformation as a result of the Last Chance Thrust. This thrust developed in the Mesozoic, probably in the Jurassic, and resulted in the emplacement of Cambrian siliceous and carbonate rocks over Mississippian shale, siltsone, and quartzite. Formations represented in the western part of the area are the Zabriski Quartzite (Lower Cambrian), Carrara (Lower and Middle Cambrian), and Perdido (Mississippian). This structurally prepared ground provided a locus for the Tertiary (Pliocene) hot spring activity and subsequent mineralization at Crater (Wrucke and others, 1984).

The mines at Crater are in large zones of sulfur-gypsum sinter that have replaced Carrara limestone and Bonanza King dolomite. These zones are centered al ong major northeast and north-south-trending faults and appear to have been the locus for the hot spring activity.

\section{METHODS OF STUDY}

\section{Sample Media}

The purpose of this study was to augment the rock sampling done during the mineral resource study of the Sal ine Valley Wilderness Study Area (Wrucke and others, 1984). Analytical data from samples collected during the examination of the study area helped in the identification of the mineral 


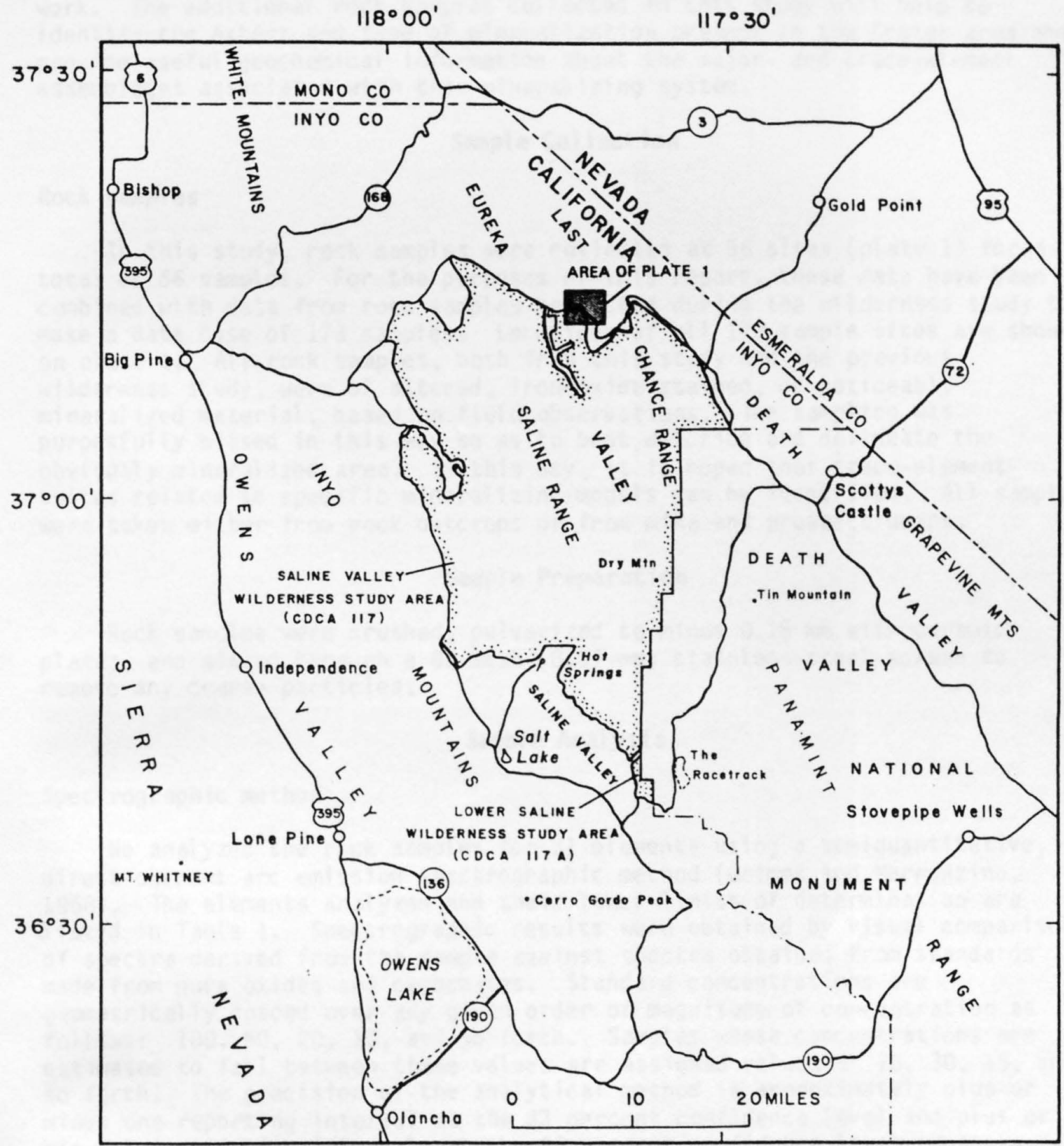

Fig. 1 -- Map showing the location of Saline Valley and Lower Saline Wilderness Study Areas and the Crater area of mercury-sulfur-gypsum mineralization (area of Plate 1), Inyo County. California. 
potential of the Crater area and al so identified the need for more detailed work. The additional rock samples collected in this study will help to identify the extent and type of mineralization present in the Crater area and provide useful geochemical information about the major-and trace-element assemblages associated with this mineralizing system.

\section{Sample Collection}

\section{Rock samples}

In this study, rock samples were collected at 56 sites (plate 1) for a total of 66 samples. For the purposes of this report, these data have been cambined with data from rock samples collected during the wilderness study to make a data base of 173 samples. Locations of all 173 sample sites are shown on plate 1. All rock samples, both from this study and the previous wilderness study, were of altered, iron-oxide stained, or noticeably mineralized material, based on field observations. The sampling was purposfully biased in this way so as to best describe and delineate the obviously mineralized area. In this way, it is hoped that trace-element suites related to specific mineralizing models can be identified. All samples were taken either from rock outcrops or from mine and prospect dumps.

\section{Sample Preparation}

Rock samples were crushed, pulverized to minus $0.15 \mathrm{~mm}$ with ceramic plates, and sieved through a 80 -mesh $(0.17-\mathrm{mm})$ stainless-steel screen to remove any coarse particles.

\section{Sample Analysis}

\section{Spectrographic method}

We analyzed the rock samples for 31 elements using a semiquantitative, direct-current arc emission spectrographic method (Grimes and Marranzino, 1968). The elements analyzed and their lower limits of detemination are listed in Table 1. Spectrographic results were obtained by visual comparison of spectra derived from the sample against spectra obtained from standards made from pure oxides and carbonates. Standard concentrations are geometrically spaced over any given order of magnitude of concentration as follows: $100,50,20,10$, and so forth. Samples whose concentrations are estimated to fall between those values are assigned values of $70,30,15$, and so forth. The precision of the analytical method is approximately plus or minus one reporting interval at the 83 percent confidence level and plus or minus two reporting intervals at the 96 percent confidence level (Motooka and Grimes, 1976). Values determined for the major elements (iron, magnesium, calcium, and titanium) are given in weight percent; all others are given in parts per million (micrograms/gram). Table 3 gives the descriptions of rock samples collected in the Crater mercury-gypsum-sulfur mineralized area. Analytical data for samples from the Crater area are listed in Table 4. 


\section{Chemical Methods}

Other methods of analysis used on samples from the Crater area are summarized in Table 2 .

Analytical results for rock samples are listed in Table 4.

\section{ROCK ANALYSIS STORAGE SYSTEM}

Upon completion of all analytical work, the analytical results were entered into a computer-based file called Rock Analys is Storage System (RASS). This data base contains both descriptive geological information and analytical data. Any or all of this infomation may be retrieved and converted to a binary form (STATPAC) for computerized statistical analysis or publication (VanTrump and Miesch, 1976).

\section{DESCRIPTION OF DATA TABLES}

Table 4 lists the analyses for the rock samples. For the table, the data are arranged so that column 1 contains the USGS-assigned sample numbers. These numbers correspond to the numbers shown on the site location map (plate 1). Columns in which the element headings show the letter "s" bel ow the element symbol are emission spectrographic analyses; "aa" indicates atomic absorption analyses. A letter " $N$ " in the tables indicates that a given element was looked for but not detected at the lower limit of determination shown for that element in table 1. If an element was observed but was bel ow the lowest reporting value, a "less than" symbol $(<)$ was entered in the tables in front of the lower limit of detemination. If an element was observed but was above the highest reporting value, a "greater than" symbol ( $>$ ) was entered in the tables in front of the upper limit of detemination. If an element was not looked for in a sample, two dashes (--) are entered in table 4 in place of an analytical value. Because of the fomatting used in the computer program that produced table 4, some of the elements listed in these tables (Fe, Mg, $\mathrm{Ca}, \mathrm{Ti}, \mathrm{Ag}$, and $\mathrm{Be}$ ) carry one or more nonsignificant digits to the right of the significant digits. The analysts did not determine these elements to the accuracy suggested by the extra zeros.

The spectrographic deteminations for $\mathrm{Au}$, Sn, and Th in the rock samples were all below the lower limits of determinations shown in Table 1; consequently, the columns for these elements have been deleted from Table 4 . 


\section{REFERENCES CITED}

Grimes, D. J., and Marranzino, A. P., 1968, Direct-current arc and alternating-current spark emission spectrographic field methods for the semiquantitative analysis of geologic materials: U.S. Geological Survey Circular 591, $6 \mathrm{p}$.

McNerney, J. J., Buseck, P. R., and Hanson, R. C., 1972, Mercury detection by means of thin gold films: Science, $v$. 178, p. 611-612.

Motooka, J. M., and Grimes, D. J., 1976, Analytical precision of one-sixth order semiquantitative spectrographic analyses: U.S. Feological Survey Circular 738, 25 p.

Thompson, C. E., Nakagawa, H. M., and Van Sickle, G. H., 1968, Rapid analysis for gold in geologic materials, in Geological Survey research 1968: U.S. Geological Survey Professional Paper 600-B, p. B130-B132.

VanTrump, George, Jr., and Miesch, A. T., 1976, The U.S. Geological Survey RASS-STATPAC system for management and statistical reduction of geochemical data: Computers and Geosciences, v. 3, p. 475-488.

Vaughn, W. W., and McCarthy, J.H., Jr., 1964, An instrumental technique for the determination of submicrogram concentrations of mercury in soils, rocks, and gas, in Geological Survey research 1964: U.S. Geological Survey Professional Paper 501-D, P. D123-D127.

Viets, J. G., 1978, Detemination of silver, bismuth, cadmium, copper, lead, and zinc in geologic materials by atomic absorption spectrometry with tricaprylylmethyl ammonium chl oride: Analytical Chemistry, v. 50, p. 1097-1101.

Ward, F. N., Lakin, H. W., Canney, F. C., and others, 1963, Analytical methods used in geochemical exploration by the U.S. Geological Survey: U.S. Geological Survey Bulletin 1152, $100 \mathrm{p}$.

Ward, F. N., Nakagawa, H. M., Harms, T. F., and Van Sickle, G. H., 1969, Atomic-absorption methods useful in geochemical exploration: U.S. Geological Survey Bulletin 1289, $45 \mathrm{p}$.

Wrucke, C. T., Marsh, S. P., Raines, G. L., Werschky, R. S., Blakely, R. J., Hoover, D. B., McHugh, E., Rumsey, C. M., Gaps, R. S., and Causey, J. D., 1984, Mineral resource potential of the Sal ine Valley and Lower Sal ine Valley Wilderness Study Areas, Inyo County, California: U.S. Geological Survey Open-File Report 84-560, 72 p. 
TABLE 1.--Limits of determination for the spectrographic analysis of rocks and stream sediments, based on a 10-mg sample

[The spectrographic limits of determination for heavy-mineral-concentrate samples are based on a 5 -mg sample, and are therefore two reporting intervals higher than the limits given for rocks and stream sediments]

El ements

Lower determination limit

Upper determination limit

Percent

Iron ( $\mathrm{Fe})$

Magnesium (Mg)

Calcium ( $\mathrm{Ca})$

Titanium ( $\mathrm{Ti}$ )
0.05

.02

.05

.002
20

10

20

Parts per million

Manganese (Mn)

Silver ( $\mathrm{Ag})$

Arsenic (As)

Gold ( $A u$ )

Boron (B)

Barium ( $\mathrm{Ba}$ )

Beryllium (Be)

Bi smuth ( $B i$ )

Cadmium (Cd)

Cobalt (Co)

Chromium (Cr)

Copper (Cu)

Lanthanum (La)

Mol ybdenum (Mo)

Niobi um ( $\mathrm{Nb}$ )

Nickel ( $\mathrm{Ni}$ )

Lead $(P b)$

Ant imony ( $\mathrm{Sb}$ )

$\mathrm{Sc}$ and $\mathrm{i} u \mathrm{~m}$ (SC)

Tin (Sn)

Strontium (Sr)

Vanadium (V)

Tungsten ( $W$ )

Yttrium ( $Y$ )

Zinc $(\mathrm{Zn})$

Zirconium ( $\mathrm{Zr}$ )

Thorium (Th)
10

0.5

200

10

10

20

1

10

20

5

10

5

20

5

20

5

10

100

5

10

100

10

50

10

200

10

100

$$
\begin{array}{r}
5,000 \\
5,000 \\
10,000 \\
500 \\
2,000 \\
5,000 \\
1,000 \\
1,000 \\
500 \\
2,000 \\
5,000 \\
20,000 \\
1,000 \\
2,000 \\
2,000 \\
5,000 \\
20,000 \\
10,000 \\
100 \\
1,000 \\
5,000 \\
10,000 \\
10,000 \\
2,000 \\
10,000 \\
1,000 \\
2,000
\end{array}
$$


Table 2.--Commonly used chemical methods

$$
\begin{aligned}
{[A A} & =\text { atomic absorption } ; I=\text { instrumental } \\
S & =\text { spectrographic; } C X=\text { colorimetric }]
\end{aligned}
$$

Element or constituent determined

Gold ( $\mathrm{Au}$ )

AA

0.05

T. A. Roemer and

J. D. Sharkey

Mercury $(\mathrm{Hg})$

I

0.02

10

5 or 10

Antimony (Sb)

$A A$

2 do

do

do

do

Thompson and others, 1968

$\frac{\text { Modification }}{\text { of McNerney }}$ and others, 1972, and Vaughn, and McCarthy, 1964.

Ward and others 1963

$\frac{\text { Modification of }}{\text { Viets, } 1978}$

$\frac{\text { Modification of }}{\text { Viets, } 1978}$ and Ward and others, 1969
$A A$

$A A$

AA

AA
5

0.1

5

.05 do

do

do

do-.-..-.-

do
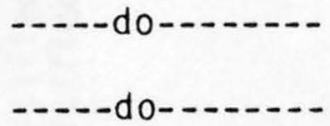

Silver $(A g)$ 
Table 3. -- Descriptions of rock samples collected in the Crater mercury-gypsum-sulfur mineralized area, Inyo County, California

CR001

CRO02

CROO3

CR004

CR005

CR006

CR007

CR008

CR007A

CR009

CR010

CR011

CR012

CR012A

CR013

CR014

CR014A

CR015

CR015A

CR016

CR017

CR018

CR019

CR020

CR021

CR022

CRO23

CR024

CR025

CR026
Gypsum and soft red iron oxides

Quartzite, jasperoid, breccia

Carrara Fm., silicified, iron oxide stained shale

Gypsum and soft iron oxides from prospect pit

Carrara Fm., dark-red-brown silicified limestone

Bonanza King Fm., gypsum intermixed with soft dark-red-brown iron oxides

Bonanza King Fm., Brecciated gypsum intermixed with light-brown iron oxides

Bonanza King Fm., silicified dolomite with iron oxides

Bonanza King Fm., brecciated and iron oxide stained dolomite

Bonanza King Fm., dolomite replaced by gypsum and iron oxides

Carrara Fm., fine grained, silicified and finely brecciated, dark-red-brown limestone Carrara Fm.(?), limestone, brecciated and stained with iron oxides

Carrara Fm.(?), limestone drill cuttings, unaltered

Alluvium, gypsum and iron oxide cemented

Nopah Fm., fault gouge, red-brown iron oxides

Nopah Fm., fractured and iron oxide stained dolomite

Nopah Fm., leached and iron oxide stained dolomite

Carrara Fm., red to red-brown silicified and brecciated limestone

Carrara Fm., white sinter(?) nodules in silicified limestone

Carrara Fm., red, fine-grained, silicified limestone, with opaline nodules

Carrara Fm., red silicified limestone

Carrara Fm., brown silicified limestone with opaline quartz

Nopah Fm., dark-brown gossan with gypsum from fault zone

Nopah Fm. (Dunderberg Shale), bleached, containing iron oxides and gypsum

Nopah Fm. (Dunderberg Shale), limy, stained brown to yellow-brown, fractured

Perdido Fm., silicified shale with quartz veins, sulfur, and gypsum

Perdido Fm., silicified shale with iron and manganese oxides

Perdido Fm., intensly silicified shale, with iron oxides and alunite on fractures

Perdido Fm., silicified shale with brown iron oxides and some red jasperoid

Perdido Fm., silicified chert-pebble conglomerate with yellow-green stains 
CR027

CR028

CR029

CR030

CR031

CR032

CR033

CR034

CR035

CR036

CR037

CR038

CR039

CR040

CR041

CR041A

CR041B

CR042

CR0 43

CR043A

CR044

CR045

CR046

CR047

CR0 48

CR049

CR049A

CR050

CR051

CR052
Zabriski Quartzite, massive quartzite and quartz with some yellowish stains Perdido Fm., silicified shale with brown iron oxides and small quartz veins Perdido Fm., silicified shale, with brown iron oxides and yellowish stains on fractures Perdido Fm., silicified and fractured shale with red and dark brown iron oxides Perdido Fm., silicified shale with yellowish-green stains and brown iron oxides

Perdido Fm., black iron and manganese oxides, from thrust zone, siliceous Perdido Fm., tan to brown jasperoid fracture filling with iron oxides Zabriski Quartzite, fractured and cemented with iron oxides, silica, and gypsum Nopah Fm., fractured and brecciated dolomite with red iron oxides

Nopah Fm., silicified and fractured dolomite with iron oxides on fractures

Nopah Fm., shear zone in dolomite with iron oxides and secondary calcite veins Nopah Fm., recrystalized dolomite with red iron oxides

Nopah Fm., brown calcite vein in recrystalized dolomite, some iron oxides

Nopah Fm. (Dunderberg Shale), from fault zone, sheared and broken, abundant red iron oxides Bonanza King Fm., silicified dolomite with some gypsum

Bonanza King Fm., gossan, dark-red-brown, abundant iron oxides

Bonanza King Fm., red and brown jasperoid

Bonanza King Fm., red silicified dolomite

Rest Spring Fm.(?), red silicified shale

Tertiary conglomerate, light-brown, some jasperoid cement

Rest Spring Fm., recrystalized limestone with red-brown iron oxides and gypsum Rest Spring Fm., recrystalized limestone and shale, red-brown iron oxides

Zabriski Quartzite, vuggy, with dark-brown iron oxides

Zabriski Quartzite, fractured and brecciated with brown iron oxides

Zabriski Quartzite, brown iron oxide gossan from fracture zone

Zabriski Quartzite, iron oxide stained and some yellowish stains, from adit

Zabriski Quartzite, quartz vein with yellow-green stains

Zabriski Quartzite, brown iron oxide gossan, from shear zone

Perdido Fm., sheared and fractured chert, brown to red-brown iron oxide

Perdido Fm., fractured and silicified chert with yellow-green stains 
Table 3. -- Continued

CR052A

CR053

CR054

CR055

CR056

IV007R

IV019R

IV019RA

I V019RB

IV022R

IV022RA

IV022RB

I V $022 R C$

IV022RD

IV022RE

V022RF

IV $023 R$

IV023RA

I V023R B

IV $023 R C$

I V023RG

I V023R I

I V 023RJ

IV023RK

I V023RL

I V023RM

I V023R N

I V023R0

IV 023RP

IV023RQ

Perdido Fm., brown iron oxide fracture filling

Perdido Fm., fractured chert with iron oxides and yellow stains

Carrara Fm., fractured, thin-bedded shale and quartzite, with iron oxides

Carrara Fm., yellow and brown iron oxide gouge

Zabriski Quartzite, iron oxide fault gouge with gypsum, from thrust zone

Massive sulfur and gypsum

Red iron oxide gossan from Last Chance Thrust

Brown iron oxide gossan

Quartzite with secondary copper staining

Zabriski Quartzite, silicified, dark gray, from mine dump

Silicified shale with quartz veins and cinnabar

Silicified chert and shale with brown iron oxides

Silicified chert, fractured and brecciated, brown iron oxides

Brown iron oxide gossan from fault zone

Black iron oxide stained shale

Silicified shale with brown iron oxides

Breccia with quartz veins and brown iron oxides

Quartz vein

Quartzite with brown iron oxides

Quartzite with brown iron oxides and some sulfide minerals

Quartzite and dark, banded chert with iron and manganese oxide stains Banded-chert breccia

Quartzite, sheared with iron oxides and clay in fractures

Quartzite, red iron oxide stained, from shear zone

Quartzite and shale, silicified and bleached

Quartzite, iron oxide stained with some secondary copper minerals

Gossan, black iron and manganese oxide

Chert breccia, fractured, with some clay

Clay, silicified, fractured, with iron oxides

Breccia, silicified, yellow iron oxide stained 
Table 3. -- Continued

IV023RR Quartzite, silicified, brown iron oxides

IV023RS

IV023RT

IV $023 R U$

IV023RV

IV023RW

IV023RX

IV350R

IV351R

IV $351 R A$

IV $352 R$

IV 352RA

IV $353 R$

IV $354 R$

IV $364 R$

IV $371 R$

IV371RA

IV $371 R B$

IV $371 R C$

IV $371 R E$

IV $371 R F$

IV $372 R$

IV $375 R$

IV $375 R A$

IV $375 R B$

Quartzite, silicified and fractured, relic pyrite, and calcite fracture fillings

Limestone, red iron oxide stained

Chert, pervasive secondary copper staining, fractured, with clay in fractures

Dolomite, silicified, red iron oxides, jarosite on fracture surfaces

IV $375 R C$

IV $375 R D$

IV $375 R E$

IV $375 R F$

IV $375 R G$

Quartzite, bleached, red and yellow iron oxide stained

Zabriski Quartzite, basal conglomerate, red iron oxide stained

Breccia, iron oxide stained

Breccia, iron oxide stained, from near Last Chance Thrust zone

Quartzite, iron oxide stained

Quartzite, iron oxide stained

Quartzite cobble with quartz veins and iron oxide staining

Quartzite, iron oxide stained

Quartzite, sheared and iron oxide stained

Quartzite, iron oxide stained

Dolomite, dark gray with some iron oxide stains

Argillite with small quartz veins, veins are iron oxide stained and have relic pyrite Argillite, black-gray with clay

Zabriski Quartzite, with yellow iron oxide stains and pyrite in dark-gray veinlets

Zabriski Quartzite, stockwork quartz veins with pyrite crystals

Zabriski Quartzite, brecciated, iron and manganese oxide stained, relic pyrite

Shale, red iron oxide stained, with quartz-calcite and quartz veins

Tuff, water laid, white, coarse grained, and crossbedded

Volcanic conglomerate, brown and black iron and manganese stained

Shale, thin bedded with abundant oxidized pyrite, red weathering

Shale, gray-green, many quartz veins with oxidized pyrite

Limestone-dolomite conglomerate, red iron oxide stained

Shale, gray-green, some oxidized pyrite

Zabriski Quartzite, brecciated, some fine-grained sulfide minerals

Owl Canyon Fm., shale, red and green, thin bedded 
Table 3. -- Continued

IV375RH Owl Canyon Fm., sandstone, fine grained, with fine-grained sulfide minerals

IV375R I

IV $375 R \mathrm{R}$

IV375RK

IV $375 R L$

Owl Canyon Fm., shale, iron oxide stained

Quartz veins, galena and pyrite bearing

Dolomite

IV $375 R M$

IV $375 R \mathrm{~N}$

IV $375 R 0$

IV $376 R$

IV $376 R A$

Dolomite, galena bearing

IV376RB

IV $376 R C$

IV376RD

IV376RE

IV376RF

Dolomite breccia, yellow iron oxide stained

Black shale, abundant calcite veinlets

Conglomerate, siliceous, black

Rest Spring Fm., shale with slight iron oxide staining

Rest Spring Fm., shale, silicified, red iron oxide stained

IV376RG

IV $376 R H$

IV376RI

IV $376 R J$

IV $376 R K$

Rest Spring Fm., silicified shale, fractured

Quartz vein, red iron oxide stained

Rest Spring Fm., silicified shale

Breccia, silicified, minor iron oxide staining

Dolomite, fractured, brown iron oxide stain on fractures

IV $376 R L$

IV376RM

IV $377 R$

IV $377 R A$

Quartzite, iron oxide stained on fractures

Limestone, iron oxide stained calcite veins

Perdido Fm.(?), shale with iron oxide stained quartz veins

Gossan in dolomite, brown and black iron oxides

Dolomite, yellow iron oxide stained

IV377RB

Concretion in dolomite, brown iron oxide stained Shale, iron oxide stained

Limestone, brown-yellow iron oxide stained, some brown cacite veins

Dolomite, red iron oxide stained, some pyrite casts

Dolomite, red iron oxide stained and brecciated

IV $377 R C$

IV $377 R D$

IV $377 R E$

IV377RF

IV377RG

Calcite vein, fractured and red iron oxide stained

Limestone, oxidized pyrite and iron oxide stained

Limestone, totally stained with brown iron oxide

Limestone with abundant oxidized pyrite

Dolomite with white dolomite veins 
Table 3. -- Continued

$\begin{array}{ll}\text { IV389R } & \text { Zabriski Quartzite, fractured and bleached } \\ \text { IV389RA } & \text { Gossan, red and brown iron oxides } \\ \text { IV389RB } & \text { Limestone, red and yellow iron oxide stained } \\ \text { IV400RA } & \begin{array}{l}\text { Gypsum-sinter } \\ \text { Gypsum-cinnabar ore from El Capitan mine }\end{array} \\ \text { IV400RB } & \text { Brecciated dolomite from El Capitan Mine } \\ \text { IV400RC } & \text { Fault gouge, brown iron oxides } \\ \text { IV400RD } & \text { Gypsum-sulfur-sinter at Crater, channel sample } \\ \text { IV400RR } & \text { Zabriski Quartzite, iron oxide stained } \\ \text { SV317R1 } & \text { Zabriski Quartzite, black iron or manganese oxide stained } \\ \text { SV317R2 } & \text { Zabriski Quartzite, yellow iron oxide stained } \\ \text { SV321R1 } & \text { Zabriski Quartzite, brecciated and iron oxide stained } \\ \text { SV321R2 } & \text { Gypsum(?) } \\ \text { SV322R1 } & \text { Shale, carbonaceous } \\ \text { SV322R2 } & \text { Zabriski Quartzite, stockwork veining with some sulfides } \\ \text { SV322R3 } & \text { Quartz vein, brecciated } \\ \text { SV334R1 } & \text { Chert-jasperoid, gray and white } \\ \text { SV334R2 } & \text { Chert-jasperoid, brown yellow } \\ \text { SV334R3 } & \text { Chert, red, with siltstone } \\ \text { SV334R4 } & \text { Chery, yellow gray, with quartz veins } \\ \text { SV334R5 } & \\ \text { SV334R6 } & \text { Chert, olive green, with quartz veinlets } \\ \text { SV334R7 } & \text { Chert, gray white, massive }\end{array}$


Table 4 -- Analyses of rozk samples collected from the Crater mercury-sulfur-gypsum mineralized area, Inyo County,

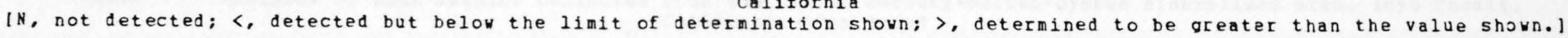

\begin{tabular}{|c|c|c|c|c|c|c|c|c|c|c|c|c|c|c|c|c|c|}
\hline Sample & L at & titu & ade & Long & itu & $d e$ & $\begin{array}{c}\mathrm{Fe}-\mathrm{pct} \\
\mathrm{s}\end{array}$ & $\begin{array}{c}M g-p c t \\
s\end{array}$ & $\begin{array}{c}\mathrm{Ca}-\mathrm{pct} . \\
\mathrm{s}\end{array}$ & $\begin{array}{c}\text { T1-pct. } \\
\mathrm{s}\end{array}$ & $\begin{array}{c}M n-p p m \\
s\end{array}$ & $\begin{array}{c}A g-p p m \\
s\end{array}$ & $\begin{array}{c}\text { As-ppm } \\
s\end{array}$ & $\begin{array}{c}B-p p m \\
S\end{array}$ & $\begin{array}{c}\mathrm{Ba}-\mathrm{ppn} \\
\mathrm{s}\end{array}$ & $\begin{array}{c}\mathrm{Be}-\mathrm{pp} \\
5\end{array}$ & $\begin{array}{c}\mathrm{B} 1-\mathrm{PD} \mathbb{A} \\
\mathrm{S}\end{array}$ \\
\hline CROD 1 & 37 & 12 & 20 & 117 & 41 & 23 & 1.00 & .10 & 10.00 & .100 & 70 & $\mathrm{~N}$ & $\mathrm{~N}$ & 100 & 200 & 1.0 & N \\
\hline $\mathrm{CROO} 2$ & 37 & 12 & 23 & 117 & 41 & 24 & .50 & .20 & 2.00 & .500 & 500 & $\mathbb{N}$ & $\mathrm{N}$ & 70 & 5,000 & $<1.0$ & $\mathrm{~N}$ \\
\hline CRวग3 & 37 & 12 & 21 & 117 & 41 & 19 & .50 & $>10.00$ & $>23.00$ & .050 & 100 & $\mathrm{~N}$ & $N$ & 70 & 100 & $<1.0$ & $\mathrm{~N}$ \\
\hline CROO 4 & 37 & 12 & 22 & 117 & 41 & 17 & 1.00 & 2.00 & $>20.00$ & .150 & 300 & $\mathrm{~N}$ & $\mathrm{~N}$ & 70 & 200 & $<1.0$ & N \\
\hline CR005 & 37 & 12 & 22 & 117 & 41 & 6 & 5.00 & .30 & 10.00 & .500 & 30 & $\mathrm{~N}$ & $\mathrm{~N}$ & 100 & 300 & $<1.0$ & N \\
\hline CRDO & 37 & 12 & 42 & 117 & 41 & 7 & 7.00 & .10 & .05 & .200 & 50 & N & $\mathrm{N}$ & 100 & 500 & $1 . c$ & N \\
\hline CROO 7 & 37 & 12 & 33 & 117 & 41 & 2 & 1.00 & .05 & 10.00 & .005 & 20 & $\mathrm{~N}$ & $\mathrm{~N}$ & 50 & $<20$ & 1.0 & N \\
\hline CR007A & 37 & 12 & 33 & 117 & 41 & 2 & .30 & .10 & 10.00 & .005 & 50 & N & $\mathrm{N}$ & 70 & $\mathrm{~N}$ & 1.0 & i \\
\hline CF $ว \supset 8$ & 37 & 12 & 38 & 117 & 41 & 2 & 1.00 & 10.00 & $>20.00$ & .030 & 500 & $\mathrm{~N}$ & $<200$ & 50 & 100 & $<1.0$ & $\mathrm{y}$ \\
\hline CROOS & 37 & 12 & 48 & 117 & 41 & 2 & 2.00 & .05 & 5.00 & .100 & 20 & N & 200 & 100 & 200 & 1.0 & N \\
\hline CRO 010 & 37 & 12 & 48 & 117 & 41 & 9 & 1.00 & .10 & 1.50 & .200 & 50 & N & $\mathrm{N}$ & 150 & 500 & 1.0 & $\mathrm{~N}$ \\
\hline C.P. 011 & 37 & 12 & 58 & 117 & 41 & 5 & .70 & .70 & $>20.00$ & .020 & 500 & N & $\mathrm{N}$ & 20 & 20 & $\mathrm{~N}$ & N \\
\hline CR012 & 37 & 13 & 13 & 117 & 40 & 39 & .15 & $>10.00$ & $>25.00$ & .015 & 150 & N & $\mathrm{N}$ & 20 & $\mathrm{~N}$ & $\mathrm{~N}$ & N \\
\hline CRO12A & 37 & 13 & 13 & 117 & 40 & 39 & .10 & 1.00 & 15.00 &.$\cap 20$ & 10 & N & $\mathrm{N}$ & $\mathrm{N}$ & 50 & N & N \\
\hline CE0 013 & 37 & 12 & 48 & 117 & 39 & 43 & .20 & $>10.00$ & 20.00 & $<.002$ & 100 & N & $\mathrm{N}$ & 20 & $\mathrm{~N}$ & $N$ & N \\
\hline CRO14 & 37 & 12 & 37 & 117 & 39 & 53 & .05 & $>10.00$ & 20.00 & $<.002$ & 100 & N & $N$ & $\mathrm{~N}$ & $\mathrm{~N}$ & $\mathrm{~N}$ & $\mathrm{~N}$ \\
\hline CRO14A & 37 & 12 & 38 & 117 & 39 & 52 & 1.00 & $>10.00$ & $>20.00$ & .005 & 1.000 & N & $N$ & 30 & 50 & 1.0 & $\mathrm{~N}$ \\
\hline CRO15 & 37 & 12 & 48 & 117 & 41 & 9 & 1.00 & .50 & .70 & .150 & 100 & N & $\mathrm{N}$ & 300 & 300 & 2.0 & $\mathrm{~N}$ \\
\hline CEO15A & 37 & 12 & 48 & 117 & 41 & 9 & .20 & .03 & .05 & .003 & 50 & N & $N$ & 100 & 200 & 2.0 & N \\
\hline CR016 & 37 & 12 & 42 & 117 & 41 & 12 & .70 & .50 & .50 & .150 & 150 & N & $\mathrm{N}$ & 70 & 500 & 1.0 & N \\
\hline CR0 17 & 37 & 12 & 43 & 117 & 41 & 15 & 1.00 & .07 & 1.00 & .200 & 100 & $\mathrm{~N}$ & $<200$ & 70 & 500 & $<1.0$ & $\mathrm{~N}$ \\
\hline CRO18 & 37 & 12 & 47 & 117 & 41 & 19 & 1.00 & .15 & 1.00 & $.2 \cap 0$ & 50 & N & N & 70 & 500 & $1 \cdot 5$ & N \\
\hline EKO19 & 37 & 14 & 15 & 117 & 39 & 38 & 3.00 & 2.00 & $>20.00$ & .100 & $>5,000$ & N & $<200$ & 150 & 1,500 & 1.5 & $\mathrm{~N}$ \\
\hline CEO2O & 37 & 14 & 14 & 117 & 39 & 42 & .50 & .02 & 3.00 & .150 & 20 & N & N & 10 & 300 & $<1.0$ & N \\
\hline CE $\supset 21$ & 37 & 14 & 17 & 117 & 39 & 37 & 10.00 & .50 & 3.00 & .150 & 500 & N & 1,000 & 100 & 500 & 5.0 & $\mathrm{~N}$ \\
\hline CSO 022 & 37 & 12 & 34 & 117 & 41 & 39 & .15 & .10 & .15 & .070 & 50 & N & N & 20 & 1,000 & $\mathrm{~N}$ & $\mathrm{~N}$ \\
\hline CRO23 & 37 & 12 & 41 & 117 & 41 & 45 & 10.00 & .10 & .20 & .200 & 70 & $\mathrm{~N}$ & 200 & 200 & 5.000 & 1.0 & N \\
\hline CRO24 & 37 & 12 & 37 & 117 & 41 & 48 & 3.00 & .07 & .70 & .150 & 200 & $<.5$ & 2,000 & 100 & 2,000 & 1.0 & N \\
\hline CRO2 25 & 37 & 12 & 33 & 117 & 41 & 47 & 15.00 & $<.02$ & .05 & .100 & 30 & $N$ & 500 & 100 & 500 & $\mathrm{~N}$ & N \\
\hline $\mathrm{CRO} 26$ & 37 & 12 & 31 & 117 & 41 & 53 & 1.00 & .30 & .20 & .200 & 70 & $<.5$ & N & 100 & $>5,000$ & 1.0 & $\mathrm{~N}$ \\
\hline CKO 27 & 37 & 12 & 28 & 117 & 42 & 0 & .76 & .05 & .10 & .050 & 30 & N & $\mathrm{N}$ & 20 & 1,000 & $\kappa$ & N \\
\hline $\mathrm{CPO} 28$ & 37 & 12 & 25 & 117 & 42 & 0 & 10.00 & .02 & .05 & .100 & 20 & $\mathrm{~N}$ & 700 & 70 & $>5,000$ & $1 . c$ & N \\
\hline CRO29 & 37 & 12 & 16 & 117 & 42 & 16 & 10.00 & .20 & .07 & .200 & 50 & $<.5$ & 1.000 & 200 & 5,000 & 1.0 & $\mathrm{~N}$ \\
\hline CRO30 & 37 & 12 & 23 & 117 & 42 & 21 & 15.00 & .07 & .05 & .070 & 100 & $\mathrm{~N}$ & 500 & 150 & 2,000 & 2.0 & s \\
\hline CRO31 & 37 & 12 & 24 & 117 & 42 & 23 & 5.00 & .15 & $\cdot 10$ & .100 & 100 & N & 2.000 & 100 & 2,000 & 1.0 & $\kappa$ \\
\hline $\mathrm{CPO} 32$ & 37 & 12 & 28 & 117 & 42 & 18 & 20.00 & .20 & .20 & .100 & 1,000 & $\mathrm{~N}$ & 500 & 200 & 2.300 & 5.0 & $\mathrm{~N}$ \\
\hline $\mathrm{CRO}_{3} 3$ & 37 & 12 & 27 & 117 & 42 & 19 & 15.00 & .03 & .05 & .020 & 15 & N & 500 & 50 & 500 & $\mathrm{~N}$ & N \\
\hline CF.034 & 37 & 12 & 8 & 117 & 42 & 17 & 3.00 & .10 & 10.00 & .050 & 700 & N & 700 & 50 & 200 & $<1.0$ & N \\
\hline CRO35 & 37 & 12 & 21 & 117 & 40 & 26 & 5.00 & 5.00 & 10.00 & .200 & 500 & N & $\mathrm{N}$ & 100 & 200 & N & N \\
\hline CR036 & 37 & 12 & 18 & 117 & 40 & 17 & 1.00 & $>10.00$ & 15.00 & .050 & 1,000 & N & $\mathrm{N}$ & 50 & 20 & N & N \\
\hline $\mathrm{CRO} 37$ & 37 & 12 & 28 & 117 & 40 & 18 & .10 & $>10.00$ & 25.00 & .010 & 500 & $\mathrm{~N}$ & $\mathrm{~N}$ & $<10$ & N & N & $\mathrm{N}$ \\
\hline CR038 & 37 & 12 & 32 & 117 & 40 & 14 & 1.00 & 2.00 & $>20.00$ & .100 & 5,000 & $N$ & $\mathrm{~N}$ & 200 & 300 & $\mathrm{~N}$ & N \\
\hline CRO39 & 37 & 13 & 43 & 117 & 39 & 30 & 1.50 & 1.00 & $>20.00$ & .100 & 700 & $\mathrm{~N}$ & $N$ & 100 & 300 & N & $\mathrm{N}$ \\
\hline CRO39A & 37 & 13 & 43 & 117 & 39 & 30 & 10.00 & 2.00 & 25.00 & .150 & 1,000 & N & 200 & 150 & 200 & 1.0 & N \\
\hline CRO4O & 37 & 13 & 9 & 117 & 39 & 32 & 5.00 & 1.00 & 10.00 & .500 & 1,000 & N & $N$ & 200 & 500 & 1.5 & N \\
\hline
\end{tabular}


Table 4 -- Analyses of rock samples collected from the Crater mercury-sulfur-gypsum mineralized area, Inyo County, California--Continued

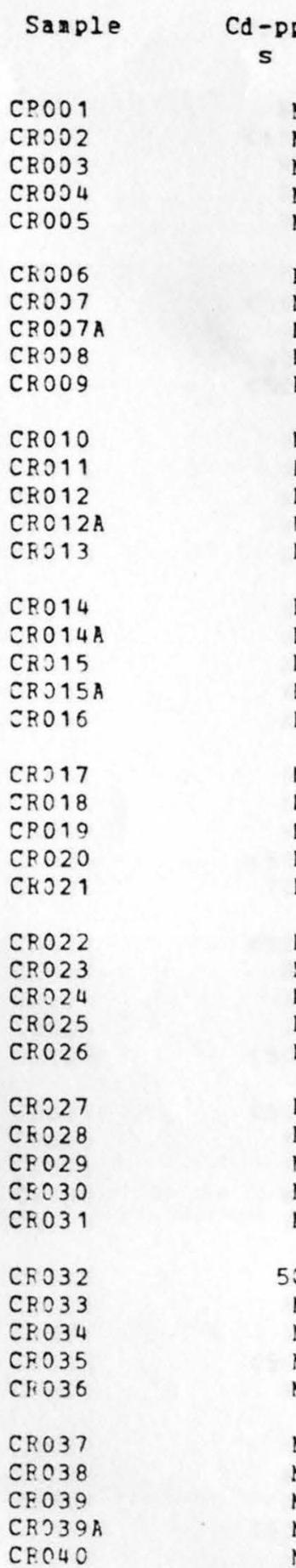

Co-ppm $\mathrm{Cr}$

s

\section{0}

$\mathrm{N}$
5
$\mathrm{~N}$
5
$\mathrm{~N}$

70
30
15
20
15
20
20
20
15
20

u-ppm

La

50
30
20
100
10

$N$
$N$
$N$
$N$

10

50
15
50

50
10

10
20

$\mathrm{N}$
$\mathrm{N}$
$\mathrm{N}$
$\mathrm{N}$
$\mathrm{N}$

70
10
15
30
30

70
50
7

7
7

30

20

20

30

70

100

70

20

20
30

7
7
50
70
50

70

100

70
$<5$

10
200

200
200

200
500

100

70
150

200

200

150

100

$\begin{array}{rr}20 & 100 \\ \mathrm{~N} & 150 \\ <5 & 10 \\ 5 & 30 \\ 7 & 15\end{array}$

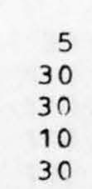

7

7
20
30
100
15

15

100
5
5
20
7

$N$
7
7
20
7

20
20
20
20
100

7
15
15
30
20
5

Mo-ppm

Nb-ppm$$
\text { s }
$$

N1-pp

$s$

-ppm

$<5$
$<5$
5
$\mathrm{~N}$

$N$
$<20$

N

$<20$

$\mathrm{N}$
$\mathrm{N}$
$\mathrm{N}$
$\mathrm{N}$
$\mathrm{N}$

$\mathrm{N}$

N

N

N$$
<20
$$

N

N$$
\begin{array}{r}
N \\
N \\
5 \\
<5 \\
10
\end{array}
$$$$
\begin{aligned}
& \mathrm{N} \\
& \mathrm{N} \\
& \mathrm{N}
\end{aligned}
$$$$
\begin{array}{r}
15 \\
7 \\
5 \\
N \\
7
\end{array}
$$$$
\begin{array}{r}
\mathrm{N} \\
50 \\
\mathrm{~N} \\
\mathrm{~N} \\
220
\end{array}
$$$$
\begin{array}{r}
<5 \\
30 \\
20 \\
<5 \\
5
\end{array}
$$$$
\begin{array}{r}
\mathrm{N} \\
\mathrm{N} \\
\mathrm{N} \\
\mathrm{N}
\end{array}
$$$$
\begin{aligned}
& 7 \\
& <5 \\
& <5 \\
& <5 \\
& <5
\end{aligned}
$$

$\begin{array}{rr}\mathrm{N} & 5 \\ \mathrm{~N} & 7 \\ \mathrm{~N} & 70 \\ \mathrm{~N} & <5 \\ <20 & 100\end{array}$

$N$
$N$
$N$
$N$
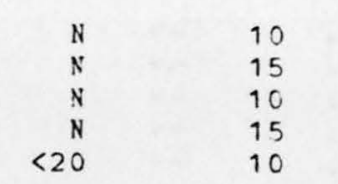

$\mathrm{N}$
$\mathrm{N}$
$\mathrm{N}$
$\mathrm{N}$$$
\begin{array}{r}
7 \\
10 \\
5 \\
200 \\
7 \\
500 \\
10 \\
70 \\
15 \\
15
\end{array}
$$$$
\begin{array}{r}
200 \\
10 \\
20 \\
15 \\
\mathrm{~N}
\end{array}
$$

$\mathrm{N}$
$\mathrm{N}$
$\mathrm{N}$
$\mathrm{N}$
$\mathrm{N}$

\begin{tabular}{l}
7 \\
5 \\
$N$ \\
7 \\
\hline 5
\end{tabular}

$<10$
$<10$
10
15
100
20
$N$
$<10$
50
70
30
$N$
$N$
$<10$
$<10$

$<10$

20

$<10$

30

30
20
30
$\mathrm{~N}$
50

20
$\mathrm{~N}$
50
$\mathrm{~N}$
30 b-ppm

$s$

Sc-ppm

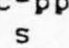

Sr-ppm

$s$

$V-p p m$

$\begin{array}{r}\text { N } \\ \text { N } \\ \text { N } \\ \text { N } \\ \hline 100\end{array}$

$<100$

$\mathrm{N}$
$\mathrm{N}$
$\mathrm{N}$
$\mathrm{N}$
$\mathrm{N}$

7
15
50
100
30

$\mathrm{N}$
50
20
15
10
70
$\mathrm{~N}$
10
20
$\mathrm{~N}$
$\mathrm{~N}$
20
15
30
10

7
5
$N$
5
5
10
$N$
5
$N$
$<5$

$\begin{array}{rr}500 & 200 \\ 150 & 50 \\ 100 & 30 \\ 200 & 30 \\ 150 & 50 \\ 500 & 150 \\ 5100 & 50 \\ 100 & 150 \\ 100 & 50 \\ 700 & 150 \\ & \\ 500 & 150 \\ 200 & 15 \\ <100 & 10 \\ N & 30 \\ N & 10\end{array}$

102

100

10

200
300

$<12$

100

15
70

$\begin{array}{rr}700 & 300 \\ 500 & 100 \\ 100 & 20 \\ N & 20 \\ 500 & 150 \\ <100 & 100 \\ 700 & 1,200 \\ 100 & 500 \\ 200 & 100 \\ 300 & 200\end{array}$

$\begin{array}{rr}200 & 20 \\ 200 & 300\end{array}$

$200 \quad 500$

200

$<100$

500
200

$\begin{array}{rr}200 & 3,000 \\ 150 & 150 \\ 100 & 200 \\ 300 & 50 \\ 100 & 20\end{array}$

$<100$

100

100
100

150

10
10
30
150
100 
Table 4 -- Analyses of rock samples collected from the Crater mercury-sulfur-gypsum mineralized area, Inyo County, California--Continued

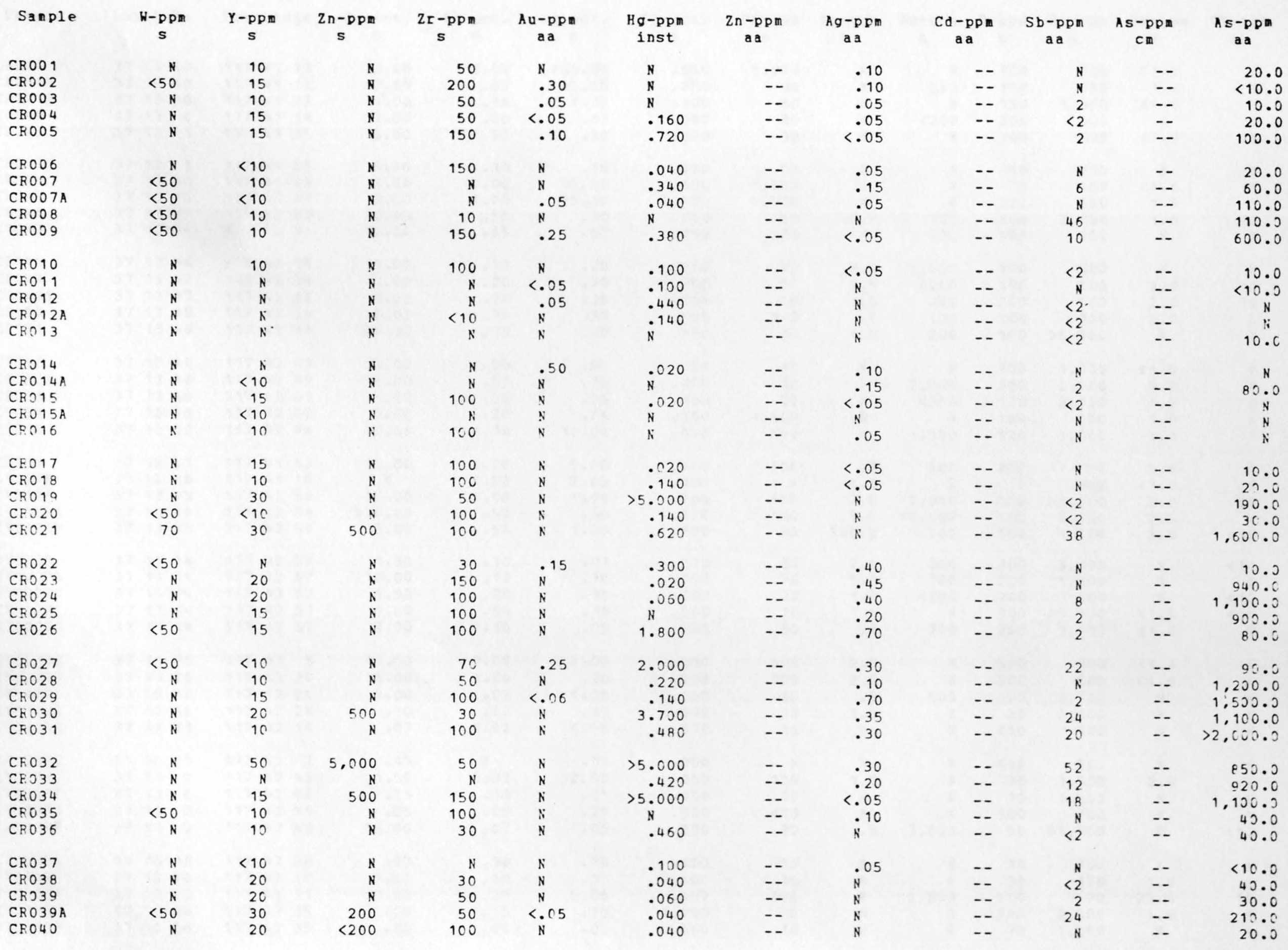


Table 4 -- Analyses of rock samples collected from the Crater mercury-sulfur-gypsum mineralized area, Inyo County, California--Continued

Sample

Latitude

CRO4 1

CRO41A

CRO4 $1 \mathrm{~B}$

$\mathrm{CRO} 42$

CF 243

CRO43A

CRO 44

ChO45

CRO4 6

CRO 047

CKO 48

Cह 049

CRO 49 A

CRO50

CR:5 1

CR 52 A

CP. 053

CPO5 4

C. 255

Ch056

IVOJ $7 \mathrm{E}$

IVO19R

IVO19R A

IVO19RB

IV $22 \mathrm{R}$

IVO22Rh

IV $022 \mathrm{~PB}$

IVO22RC

I VO $22 \mathrm{RD}$

I VO $22 \mathrm{RE}$

IV $022 \mathrm{RF}$

I $223 \mathrm{R}$

IVO2 $3 \mathrm{RA}$

IV $023 R B$

IV $223 \mathrm{RC}$

IV $023 R G$

IVO $23 \mathrm{EI}$

IVO $23 \mathrm{RJ}$

I VO2 3RK

I VO $23 \mathrm{RL}$

I V $23 \mathrm{RK}$

IVO2 3R N

IVO23RJ

I VO $23 R \mathrm{P}$

$\begin{array}{lll}37 & 13 & 1\end{array}$

$37 \quad 13 \quad 10$

$\begin{array}{llll}37 & 13 & 13\end{array}$

$\begin{array}{lll}37 & 13 & 47\end{array}$

$37 \quad 13 \quad 46$

$37 \quad 13 \quad 44$

$\begin{array}{lll}37 & 13 & 47\end{array}$

$\begin{array}{lll}37 & 13 & 47\end{array}$

71348

$37 \quad 13 \quad 48$

$\begin{array}{lll}37 & 13 & 48\end{array}$

371348

371348

371348

$\begin{array}{lll}37 & 12 & 21\end{array}$

$\begin{array}{lll}37 & 12 & 45\end{array}$

$3713 \quad 45$

$37 \quad 1346$

371346

$37 \quad 11 \quad 14$

$37 \quad 11 \quad 14$

$37 \quad 11 \quad 14$

$37 \quad 11 \quad 14$

$\begin{array}{lll}37 & 11 & 26\end{array}$

371126

$\begin{array}{lll}37 & 10 & 48\end{array}$

$\begin{array}{llll}37 & 10 & 48\end{array}$

$37 \quad 10 \quad 45$

37110

37110

37110

$37 \quad 10 \quad 18$

$\begin{array}{lll}37 & 10 & 20\end{array}$

$\begin{array}{lll}37 & 10 & 22\end{array}$

$\begin{array}{lll}37 & 10 & 26\end{array}$

\section{$s$}

$\begin{array}{llllll}37 & 13 & 10 & 117 & 41 & 13\end{array}$

$\begin{array}{llllll}37 & 13 & 10 & 117 & 41 & 13\end{array}$

$\begin{array}{llllll}37 & 13 & 10 & 117 & 41 & 13\end{array}$

$\begin{array}{llllll}37 & 13 & 8 & 117 & 41 & 14\end{array}$

$\begin{array}{llllll}37 & 13 & 1 & 117 & 41 & 25\end{array}$

$\begin{array}{lll}117 & 41 \quad 25\end{array}$

$\begin{array}{lll}117 & 41 & 33\end{array}$

$\begin{array}{lll}117 & 41 & 47\end{array}$

$11742 \quad 53$

$117 \quad 4254$

371352

$117 \quad 4256$

$117 \quad 4258$

$\begin{array}{lll}117 \quad 42 & 58\end{array}$

1174254

$117 \quad 42 \quad 48$

$117 \quad 42 \quad 49$

$11742 \quad 4$ ?

1174251

$117 \quad 4252$

$117 \quad 42 \quad 44$

1174153

$11741 \quad 10$

$11742 \quad 58$

1174259

$11742 \quad 59$

$117 \quad 42 \quad 57$

$\begin{array}{lll}117 & 42 & 57\end{array}$

$117 \quad 42 \quad 57$

$\begin{array}{llll}117 & 42 & 57\end{array}$

$117 \quad 42 \quad 57$

$\begin{array}{lll}117 & 43 \quad 5\end{array}$

$\begin{array}{lll}117 & 43 & 24\end{array}$

$117 \quad 42 \quad 24$

$117 \quad 42 \quad 24$

$117 \quad 42 \quad 14$

$\begin{array}{lll}117 & 42 \quad 2\end{array}$

$\begin{array}{llll}117 & 42 \quad 45\end{array}$

$117 \quad 42 \quad 45$

1174245

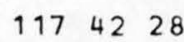

$\begin{array}{lll}117 & 42 \quad 30\end{array}$

$\begin{array}{lll}117 & 42 & 31\end{array}$

$\begin{array}{lll}117 & 42 & 35\end{array}$

$117 \quad 42 \quad 35$ mo-pet.

$\begin{array}{rrrrr}2.00 & 1.00 & >20.00 & .050 & 1,000 \\ 2.00 & .03 & 10.00 & .050 & 30 \\ 1.00 & .10 & 7.00 & .100 & 50 \\ 7.00 & .20 & .05 & .500 & 30 \\ 5.00 & .20 & .30 & .300 & 30 \\ 5.00 & .10 & .15 & .200 & 50 \\ 3.00 & 10.00 & 15.00 & .100 & 1.000 \\ 3.00 & 1.00 & 15.00 & .300 & 1.000 \\ 10.00 & .50 & .10 & .200 & 20 \\ 15.00 & .05 & .07 & .150 & 30 \\ 15.00 & .10 & .20 & .050 & 10 \\ 2.00 & .20 & .20 & .200 & 50 \\ 3.00 & .70 & .30 & .300 & 50 \\ 5.00 & .70 & .50 & .100 & 1,500 \\ 10.00 & .05 & .20 & .150 & 10 \\ 2.00 & .20 & .07 & .150 & 15 \\ 15.00 & .50 & .10 & .200 & 10 \\ 5.00 & .30 & .15 & .300 & 20 \\ 5.00 & .20 & .05 & .150 & 1,500 \\ 10.00 & 1.00 & 10.00 & .070 & 100\end{array}$

s

$-p p m$

$B-p p m$

Ba-p pm

$s$

Pe-pp

$B i-P P D$

$\begin{array}{llll}10.00 & 2.00 & 5.00 & .200\end{array}$

N

15.00

$>20.00$

5.00

1.50

10.00

1.50

1.00

7.00

10.00

15.00

2.00

.10
.07

$<.02$

.50
.50

.50

7.00

1.00

.30

1.00

.07
.10

- 10

.10
.05

.200
.100

.700

.500

.10
.10

.30
.50

.30

2.00

1.50

.02

.02
$<.02$

3.00

.20

$<.05$

.15
$<.05$

.070

.100

.100

.200

.05

1.50

.15

.05

15.00

N

5.00

.10
.05

.05
.07

.27

10. 00

.15
.20

.05

.70

.07

1.50
10.00

1.00

- 10

.10
.20

.15

.05

.07
5.00

.30

.05

.500

.700
.100

.070

.300

.050

150
.500

.300

.300

.300

1.000

.500

$\mathrm{N}$
$\mathrm{N}$
$\mathrm{N}$
$\mathrm{N}$
$\mathrm{N}$
$\mathrm{N}$
$\mathrm{N}$
$\mathrm{N}$

500

$<200$

100

150

150
200

100

150

1,000

500
500

$<1.0$

1.5

$<{ }^{N} .0$

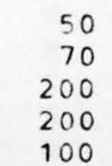

$3.0 \quad 5,000$

$<.5<200$

$3.0 \quad 200$

.5
.500
$<.5$

$$
20
$$$$
\begin{array}{r}
\text { N } \\
2,000 \\
\angle 200
\end{array}
$$

1,500

50
70

700

$<1.0$

N

200

2., 000

<. 0

1.5
1.0

$100 \quad 500$

150

200

$100>5.000$

100

$\mathrm{N}$
100
100

50

30
70

$$
\begin{aligned}
& 20 \\
& 10 \\
& 50
\end{aligned}
$$

500

500
700
30

30
30
15

15

N
150
50

$<10$

150
10
300
30

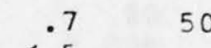

$<.5$

2.0 2,000

$300.0 \quad 10.000$

$\begin{array}{rr}2.0 & 200 \\ 5.0 & 700 \\ 1.0 & <200 \\ 1.5 & \mathrm{~N} \\ .7 & 700\end{array}$

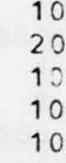

1,000

2,000

2,000

1,000 
Table 4 -- Analyses of rock samples collected from the Crater mercury-sulfur-gypsum mineralized area, Inyo County, California--Continued

Sample

CFO 41

CRO4 1A

CRO $41 \mathrm{~B}$

CRO 42

$\mathrm{CRO} 03$

CRO43A

$\mathrm{CKO} 44$

CRO 45

CRO 45

CR 047

CRO48

CRO49

CRO49A

CRO 50

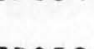

CRO52A

CRO 53

CR. 054

CRO56

IVOO 7 R

IVO $19 \mathrm{~K}$

IV019EA

IV019RE

IV $022 \mathrm{R}$

I $V 022 \mathrm{RA}$

I V0 $22 \mathrm{RB}$

IVO $22 \mathrm{RC}$

IVO $22 R D$

IVO $22 R E$

IVO22RE

IVO $23 \mathrm{R}$

IV $023 \mathrm{RA}$

IVO2

IVO2 $3 \mathrm{HC}$

I VO $23 \mathrm{RG}$

IV02 3R I

IV $023 \mathrm{RJ}$

IVO $23 R K$

I $V 023 \mathrm{RL}$

IVO2 $3 \mathrm{RM}$

IVO $23 \mathrm{RN}$

IVO $23 R O$

I VO2 3R P $\mathbf{s}$

$s$

Cr-ppm

$\mathbf{s}$

$\mathrm{Cu}-\mathrm{p}$

$s$

a $-p$

$s$

Mo-ppm

b-ppm

$s$

i-ppm

15
20

$\begin{array}{rr}50 & 20 \\ 15 & 10 \\ 150 & 100\end{array}$

70

100

100

15
30

20
20

20

50
50

50
70
15

15
100

70

50
150

100

200

70
100

100
100

20

10
100
50

50
$N$
150
30
70

$\begin{array}{rr}70 & <20 \\ 5 & 20 \\ 1,000 & 150 \\ 2,000 & 20 \\ 10,000 & 50\end{array}$

$<20$

20

20
$<5$

$<5$
$<5$

200

10

10

30

30
15

15
30

10
30
$<10$
20
10
15
50
70

50

50
20

150

20
150
30
20
30

30
50
50
70
50

50

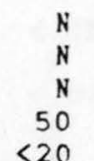

5
5
$\mathrm{~N}$
20
5

5
$\mathrm{~N}$
5
$<5$
70

$\mathrm{N}$
$<20$
50
$\mathrm{~N}$

20
100
150
15

15
20

7
100
20

20
5

30

5
1,000
2,000

100

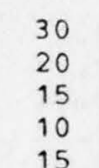

$\begin{array}{rr}50 & 100 \\ 30 & 70 \\ 7 & 50 \\ <5 & 50 \\ 10 & 50\end{array}$

10
30
10
5
15

100
$\mathrm{~N}$
$<20$
$\mathrm{~N}$
100

15
70
$<5$
30
10

$\mathrm{N}$
$\mathrm{N}$
$\mathrm{N}$
150
100

$N$
20
70
30
10
$\mathrm{N}$
$\mathrm{N}$
20

N

15

7
15

$N$

$N$
$<20$

$\mathrm{N}$

$<20$

$<20$

$<20$

$<5$
$<5$

$<5$
70
$<5$
$N$

$100^{N}$

$<20$

10

50
$<20$
$\mathrm{~N}$
100
70

$\mathrm{N}$
7
1,000
100
5

$\begin{array}{rr}N & 10 \\ N & <5 \\ N & 5 \\ <20 & 10 \\ N & 15\end{array}$

$P b-P p m$

$S$

$S b-p p m$

$$
\text { s }
$$

Sr-ppm

v-ppm

$\begin{array}{lr}\text { N } & 7 \\ \text { N } & 5 \\ \text { N } & 5 \\ \text { N } & 20 \\ \text { N } & 10\end{array}$

7
5
5
20
10

200

1.000

1,000
200

700

200

200

100

50

20
7

50
70

200
70

200

$<10$

7
7
7

15
50

$\begin{array}{rr}20 & 20 \\ <5 & 2,000 \\ 150 & 3,000 \\ 150 & 1,000 \\ 100 & 300\end{array}$

70
150
70
20
100

150
500
50

50
50

15

100
100
7
$<5$

50
100
50
20
30

30
300
30

50
300

15
$\mathrm{~N}$
70
200
70

500
$<100$
150
$\mathrm{~N}$
$\mathrm{~N}$

$<5$
7
15
10
10

700
$<100$

100

100

20

203

500

N
7000

5,000

1,000

150

.

$<100$
$<100$
700
200

$\mathrm{N}$

$\mathrm{N}$
3.000
300
200

2,000

--
5
10
5

100
502
$N$

$<5$
5
$N$
10
5

$\begin{array}{rrr}15 & 150 & 50 \\ \mathrm{~N} & \mathrm{~N} & 10 \\ 20 & 700 & 100 \\ \mathrm{~N} & \mathrm{~N} & 30 \\ 10 & \mathrm{~N} & 70\end{array}$

-.

$--$

10

500
$\mathrm{~N} \quad 150$

1,000

300

- $200 \quad 300$

$\begin{array}{lll}-- & 300 & 300\end{array}$

$--$

$<100$

10
$<12$

$<100<10$

$<100 \quad 200$

$\begin{array}{rr}302 & 50 \\ \text { N } & 30\end{array}$ 
Table 4 -- Analyses of rock samples collected from the Crater mercury-sulfur-gypsum mineralized area, Inyo county, California--Continued

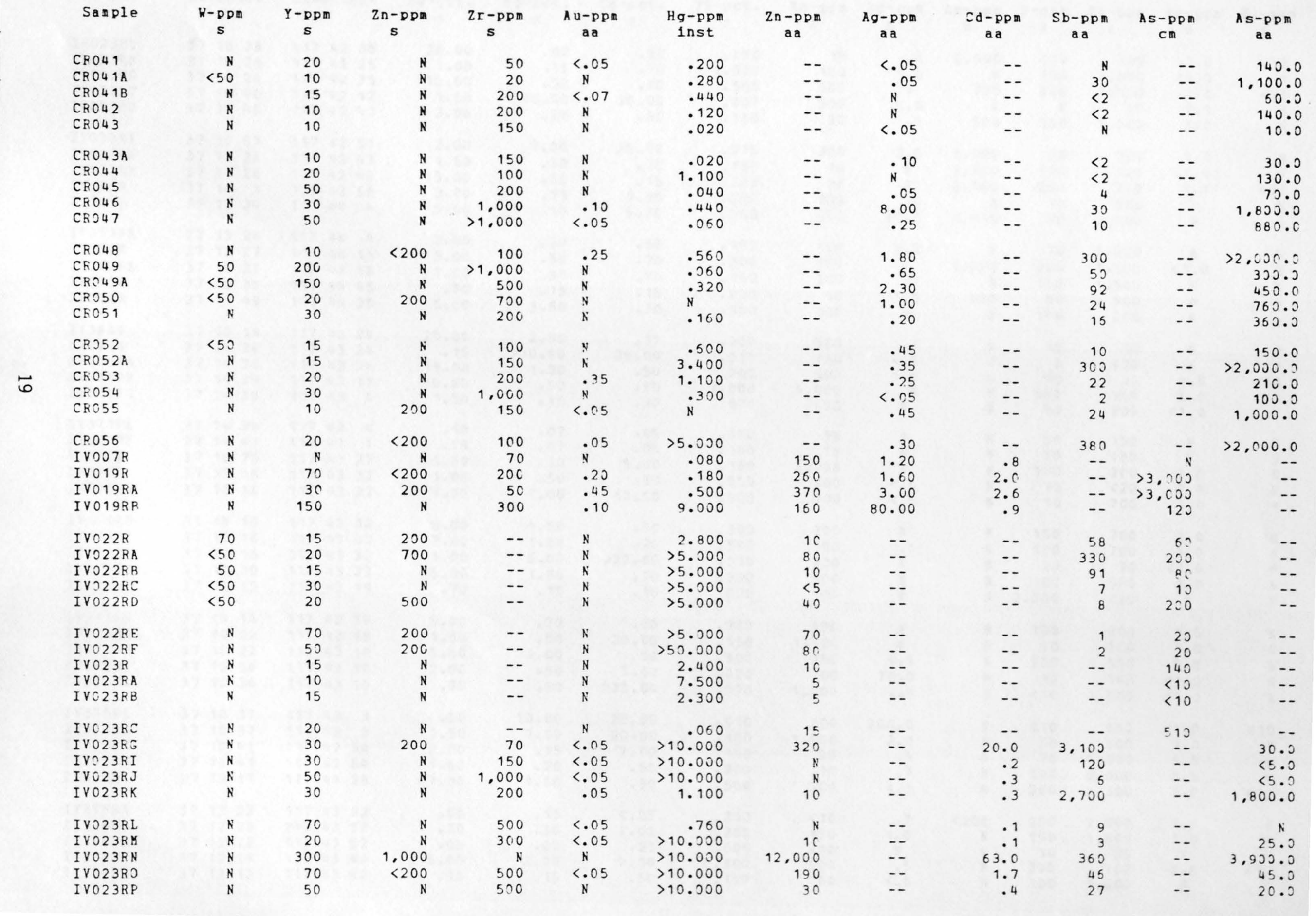


Table 4 -- Analyses of rock samples collected from the Crater mercury-sulfur-gypsum mineralized area, Inyo County. California--Continued

Sample

IVO $23 R 0$

IV $023 \mathrm{RR}$

IV $023 \mathrm{RS}$

IY023RT

IV023RU

IV $023 \mathrm{RV}$

IV $023 \mathrm{RW}$

IV023RX

IV $350 R$

IV $351 R$

IV $351 R A$

IV $352 \mathrm{R}$

IV $352 \mathrm{RA}$

IV $353 \mathrm{R}$

IV $354 R$

IV $364 \mathrm{R}$

I $371 \mathrm{R}$

N IV $371 \mathrm{R}$

IV $371 \mathrm{RE}$
IV $371 \mathrm{R}$

IV $371 \mathrm{PE}$

IV $371 \mathrm{RE}$

IV $372 \mathrm{~F}$

I $\vee 375 \mathrm{P}$

IV $375 \mathrm{RA}$

IV $375 \mathrm{RB}$

IV $375 \mathrm{RC}$

I $375 R D$

IV $375 \mathrm{RE}$

IV $375 \mathrm{KF}$

IV $375 \mathrm{kG}$

IV $375 \mathrm{RH}$

IV $375 \mathrm{RI}$

IV $375 \mathrm{RJ}$

IV $375 R K$

IV $375 \mathrm{RI}$

IV $375 \mathrm{RM}$

IV $375 \mathrm{RN}$

IV $375 \mathrm{RO}$

IV $376 R$

IV $376 \mathrm{RA}$

IV $376 \mathrm{RB}$

IV $376 \mathrm{RC}$

IV $376 \mathrm{RD}$

IV $376 \mathrm{FE}$

$\begin{array}{rrrrrr}37 & 10 & 26 & 117 & 42 & 35 \\ 37 & 10 & 26 & 117 & 42 & 35 \\ 37 & 10 & 26 & 117 & 42 & 35 \\ 37 & 10 & 40 & 117 & 42 & 42 \\ 37 & 10 & 40 & 117 & 42 & 42 \\ 37 & 10 & 57 & 117 & 42 & 51 \\ 37 & 11 & 26 & 117 & 42 & 43 \\ 37 & 11 & 26 & 117 & 42 & 43 \\ 37 & 14 & 3 & 117 & 43 & 56 \\ 37 & 13 & 24 & 117 & 44 & 4 \\ 37 & 13 & 24 & 117 & 44 & 4 \\ 37 & 12 & 27 & 117 & 44 & 18 \\ 37 & 12 & 27 & 117 & 44 & 18 \\ 37 & 12 & 36 & 117 & 44 & 45 \\ 37 & 11 & 49 & 117 & 44 & 25 \\ 37 & 10 & 19 & 117 & 43 & 28 \\ 37 & 14 & 36 & 117 & 43 & 24 \\ 37 & 14 & 36 & 117 & 43 & 24 \\ 37 & 14 & 29 & 117 & 43 & 11 \\ 37 & 14 & 38 & 117 & 43 & 4 \\ 37 & 14 & 38 & 117 & 43 & 4 \\ 37 & 14 & 41 & 117 & 43 & 1 \\ 37 & 10 & 26 & 117 & 41 & 27 \\ 37 & 10 & 16 & 117 & 43 & 32 \\ 37 & 10 & 16 & 117 & 43 & 32\end{array}$

e-pct. $s$ $s$

20.00
1.00
10.00
1.50
3.00
2.00
1.50
15.00
3.00
5.00
2.00
5.00
1.00
.70
5.00

$s$

Mn-ppm

A g-

-ppm As-ppm $\mathrm{A}-\mathrm{p} p$
$\mathrm{~s}$

$B-p P$

20.00

15.00
10.00

$\begin{array}{lll}37 & 10 & 16\end{array}$

$37 \quad 10 \quad 16$

$37 \quad 10 \quad 16$

$37 \quad 10 \quad 20$

$\begin{array}{lll}37 & 10 & 15\end{array}$

$\begin{array}{lll}37 & 12 & 15\end{array}$

$37 \quad 10 \quad 22$

$37 \quad 10 \quad 22$

$\begin{array}{lll}37 & 10 & 36\end{array}$

$\begin{array}{lll}37 & 10 & 36\end{array}$

$\begin{array}{lll}37 & 10 & 37\end{array}$

$\begin{array}{lll}37 & 10 & 37\end{array}$

$37 \quad 10 \quad 41$

$\begin{array}{llll}37 & 10 & 41\end{array}$

$\begin{array}{lll}37 & 12 & 17\end{array}$

$37 \quad 12 \quad 22$

$\begin{array}{lll}37 & 12 & 22\end{array}$

$\begin{array}{lll}37 & 12 & 22\end{array}$

$3712 \quad 18$

$37 \quad 12 \quad 12$
$117 \quad 43 \quad 32$

$\begin{array}{llll}117 & 43 \quad 32\end{array}$

$117 \quad 43 \quad 32$

$\begin{array}{lll}117 & 43 & 27\end{array}$

$117 \quad 43 \quad 19$

$117 \quad 43 \quad 18$

$\begin{array}{llll}117 & 43 \quad 18\end{array}$

$\begin{array}{lll}117 & 43 & 10\end{array}$

$117 \quad 43 \quad 10$

$\begin{array}{lll}117 & 43 \quad 3\end{array}$

$11743 \quad 3$

$117 \quad 4254$

$117 \quad 44 \quad 28$

$117 \quad 43 \quad 52$

$117 \quad 43 \quad 52$

$117 \quad 4352$

$11743 \quad 49$

1174342

.02
.15
.50
0.00

.50

.70

20.00

.50

7.00

.30

.30

.10

20.00

.20

.15

3.00
5.00

.20

.50

.30

.15
1.50

.15

1.50

2.00

$>10.00$

1.50

.50
.15

.10

.15
5.00

1.00

7.00

5.00

.050

1.000

.500

.007

.015

.150

.200

.200

.050

.50

.20

.70

.15
.30

.150

.300

.150

.020
.300

.15

20.00

.50
.30

.20

.07

.07

.30

7.00

.05

.05
5.00

.50
10.50

1.50

7.00

1.00

5.00

5.00

1.50

2.00

$\begin{array}{r}.70 \\ \hline\end{array}$

.50

1.50

1.00

7.00

.50

.05

5.00

.70

.50

.15

.70

.50
3.00

.50

.50

.70

.20
$>23.00$

.70

.10

.300

.010

.200
1.000

.070

.050

.100

.150

.050
.500

.300

.500
.070

.300

.020

.50

20.00

.50
7.00

$>20.00$

.300

.150

.020

.070

.010

7.00

20.00

20.00

15
.20

.20
1.50

. 30

.100

.100

.500

.15
.30

.30
.02

2.00

$<.05$

1.00

2.00

.50

.200

.200

.005

.300
.150

.15

$\begin{array}{rr}.5 & 2.000 \\ 1.0 & \mathrm{~N} \\ \mathrm{~N} & 700 \\ <.5 & \mathrm{~N} \\ .5 & 500\end{array}$

$3.0 \quad 1,000$

$\mathrm{N} 1,000$

N 2,000

$30.5 \quad 1,000$

50

100
300

50
300

$$
1 .
$$$$
1.0
$$$$
1,00
$$

500

150

1.000

150

15

3,000

300

700

500

200

500

500

1. 500

150

1,000

.7
$<.5$

1.5

$\mathrm{N}^{5}$

N

N

$<.5$
$<.5$

$<_{N}^{N}$

N

$300 \quad 200.0$

1.000
20

20
10
700

2.
$:$
$<$

$<10$
150

150
$<10$

500

$<10$
100

700

1,500

1,500

1,000

30

100
200

70
50

200

300
700

2.000

$70 \quad 1.000$ 
Table 4 -- Analyses of rock samples collected from the Crater mercury-sulfur-gypsum mineralized area, Inyo County,

Sample $\mathrm{Cd}-\mathrm{P}$
$\mathrm{s}$

$s$

Co-ppm
s

s

IV $023 R Q$

IV $0238 R$

I $V 023 \mathrm{RS}$

IV023RT

IV023RU

IV $023 \mathrm{EV}$

I $023 \mathrm{RH}$

I $V 023 R X$

IV $350 R$

IV $351 \mathrm{R}$

I V $351 \mathrm{RA}$

I V $352 \mathrm{R}$

IV $352 \mathrm{RA}$

IV $353 R$

IV $354 \mathrm{R}$

IV $364 \mathrm{R}$

IV $371 \mathrm{~F}$

IV $371 R A$

IV $371 R E$

I $371 \mathrm{RE}$

IV $371 \mathrm{RF}$

IV $372 \mathrm{~F}$

IV $375 \mathrm{~B}$

IV $3758 \mathrm{~A}$

IV $375 \mathrm{PR}$

I $375 \mathrm{KC}$

IV $375 \mathrm{FD}$

IV $375 \mathrm{RE}$

IV $375 \mathrm{RF}$

IV $375 \mathrm{RG}$

IV $375 \mathrm{RH}$

IV $3758 \mathrm{R}$

IV $375 \mathrm{~K} \mathrm{~J}$

IV $375 R K$

IV $375 \mathrm{RL}$

IV $375 \mathrm{R}$

IV $375 \mathrm{RN}$

IV $375 \mathrm{RO}$

IV $376 \mathrm{R}$

IV $376 \mathrm{RA}$

IV $376 \mathrm{RB}$

IV $376 \mathrm{R}=$

IV $376 R D$

IV $376 R$.

$<20$

$<5$
Cu-ppn La-ppm Ko-ppm Nb-ppm Ni-ppm

$$
s
$$$$
s
$$

$\begin{array}{rr}100 & 100 \\ 50 & 50 \\ 20 & 200\end{array}$

50

$<10$

7

300

10
30
100
100

\begin{tabular}{rr}
15 & $N$ \\
7 & $<20$ \\
15 & 70 \\
15 & 50 \\
\hline
\end{tabular}

2,000

$150 \quad 30$

150
70

70
10
15

150

100
$<10$

50

200
30

300
20
150
30
30

10
10

30

$<10$

150

70
100

30
70

$<10$

50

30
150

10

20

$<10$

30
30

70

150

100

50
$<10$

150

20
200

N

50
30

30

70
$<20$
50

50
200

N

300
150
15
15
50

5
$\mathrm{~N}$
$\mathrm{~N}$
5
50

5
$<5$

10

15

10

70
$\mathrm{~N}$
20

20
15

$\mathrm{N}$

$N$
$N$
$<5$
$N$

$N$
$N$
$N$
$<20$

$<20$
20

20

100

50
$<20$

150

N

1
$<20$
70

70
$<20$

50

70
$<20$

100

100

$<20$

$\mathrm{N}$
30
$\mathrm{~N}$

150

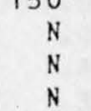

$\mathrm{N}$
$\mathrm{N}$
$\mathrm{N}$
20

$15 \quad 20$

$<20$
$\mathrm{~N}$
$\mathrm{~N}$
$<20$
$\mathrm{~N}$

$\begin{array}{rr}\mathrm{N} & 15 \\ 50 & 10 \\ 50 & 200 \\ \mathrm{~N} & 30 \\ \mathrm{~N} & 150 \\ \mathrm{~N} & 30 \\ \mathrm{~N} & <5 \\ 50 & <5 \\ \mathrm{~N} & 50 \\ \mathrm{~N} & 150\end{array}$

1,500
300

300

70

100

$<10$

15
30

500

10
100

150

30

100

50
$<5$
50

50
100
7

$<5$

$<5$
70

$<5$

30
50
7
30
10

2

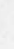

$>20,000$

150

7
10
5
20
10

$>20,000$
1,500

50

10

10
10
10
100
10
$S b-p p q$

$s$

s

Sr-ppm

$s$

$V-p p$

300

$<100$

$<100$

7
10
$N$

N

200

7.000

30

$\mathrm{N}$

洒

$\mathrm{N}$

--

20

30

N

15
$<5$

30

$<10$

1,000

$<100$

500

$<100$

500

500

$<100$

30

150

$\mathrm{N}$
$\mathrm{N}$
$\mathrm{N}$

$<5$
7
7
10
30

$<100$

N

200

300

$N$
150

$<100$

$<100$

$<100$

100

$\begin{array}{rr}N & 100 \\ N & <10 \\ N & 70 \\ 200 & 300\end{array}$

N 30

$\begin{array}{rr}N & 10 \\ N & 10 \\ N & 30 \\ 100 & <10\end{array}$

150

70

100

100

30

150

200

70

200

15

$<100 \quad 200$

$<100 \quad 100$

500

$\begin{array}{ll}500 & 150 \\ \mathrm{~F} & 100\end{array}$ 
Table 4 -- Analyses of rock samples collected from the Crater mercury-sulfur-gypsum mineralized area, Inyo County. California--Continued

\begin{tabular}{|c|c|c|c|c|c|c|c|c|c|c|c|c|}
\hline Sample & $\begin{array}{c}W-p p m \\
s\end{array}$ & $\begin{array}{c}Y-p p m \\
s\end{array}$ & $\begin{array}{c}Z n-p p m \\
s\end{array}$ & $\begin{array}{l}\text { Zr-ppm } \\
\quad s\end{array}$ & $\begin{array}{c}\mathrm{Au}-\mathrm{ppm} \\
\mathrm{a} a\end{array}$ & $\begin{array}{l}\mathrm{Hg}-\mathrm{ppm} \\
\text { Inst }\end{array}$ & $\begin{array}{c}\mathrm{Zn}-\mathrm{ppm} \\
\mathrm{a} a\end{array}$ & $\begin{array}{c}\mathrm{hg}-\mathrm{ppm} \\
\text { a a }\end{array}$ & $\begin{array}{c}C d-p p m \\
a a\end{array}$ & $\begin{array}{c}S b-p p \\
\text { a a }\end{array}$ & $\begin{array}{c}\mathrm{As}-\mathrm{ppm} \\
\mathrm{Cm}\end{array}$ & $\begin{array}{c}\text { As-ppm } \\
\text { a a }\end{array}$ \\
\hline IVO23R2 & N & 10 & N & 30 & .25 & $>10.000$ & 85 & -- & 2.2 & 360 & -- & 2.300 .0 \\
\hline IV02 3RR & N & 30 & N & 300 & .10 & $>10.000$ & 70 & -- & 2.7 & 370 & -- & 40.0 \\
\hline IV023PS & $\mathrm{N}$ & 70 & 500 & $>1.000$ & .05 & $>10.000$ & 570 & -- & .4 & 4 & -- & 270.0 \\
\hline IV023RT & N & 15 & N & 20 & .05 & $>10.000$ & 65 & -- & .9 & 41 & -- & 30.0 \\
\hline I V02 $3 \mathrm{RU}$ & N & 70 & $<200$ & 100 & .05 & $>10.000$ & 110 & -- & .7 & 48 & -- & 190.0 \\
\hline I V02 3R. V & N & 15 & $<200$ & $<10$ & $<.05$ & $>10.000$ & 140 & -- & 1.6 & 82 & -- & 330.0 \\
\hline IV023 R H & N & 15 & N & 500 & $<.05$ & .890 & $<5$ & -- & .3 & 75 & -- & 660.0 \\
\hline I VO $23 \mathrm{RX}$ & N & 30 & $\mathrm{~N}$ & 200 & $<.05$ & .130 & $<5$ & -- & .2 & 6 & -- & $1,300.0$ \\
\hline IV $350 R$ & $\mathrm{~N}$ & 20 & $<200$ & - & $\mathrm{N}$ & .070 & 130 & -- & $\cdots$ & -- & 10 & -- \\
\hline IV $351 R$ & N & 150 & $<200$ & -- & .30 & 16.000 & 70 & -- & -- & -- & 200 & -- \\
\hline IV $351 \mathrm{RA}$ & $\mathrm{N}$ & 15 & $\mathrm{~N}$ & -- & .05 & .460 & 20 & -- & -- & -- & 10 & - \\
\hline IV $352 \mathrm{R}$ & $\mathrm{N}$ & 30 & 500 & -- & $<.05$ & .420 & 150 & -- & -- & -- & 200 & - \\
\hline IV $352 \mathrm{RA}$ & $\mathrm{N}$ & 20 & 300 & -- & $N$ & .130 & 330 & -- & -. & -- & 10 & -- \\
\hline IV $353 \mathrm{R}$ & N & 10 & $N$ & -- & N & .380 & 55 & -- & -- & -- & $2 ?$ & -- \\
\hline IV $354 \mathrm{R}$ & N & 30 & K & -- & $N$ & .110 & 85 & -- & -- & -- & 10 & - \\
\hline IV $364 \mathrm{R}$ & N & 30 & 200 & -- & $\mathrm{N}$ & .090 & 190 & -- & -- & -- & 40 & - \\
\hline I V371R & N & 10 & N & $<10$ & .05 & .320 & 45 & -- & .2 & $\mathrm{~N}$ & - & $\mathrm{N}$ \\
\hline IV371RA & $\mathrm{N}$ & 30 & 300 & 150 & $<.05$ & .270 & 230 & -- & 1.2 & $N$ & -- & 20.0 \\
\hline IV $371 \mathrm{RP}$ & N & 70 & 200 & 200 & $<.05$ & .250 & 170 & -- & .8 & 2 & -- & 85.0 \\
\hline IV $371 \mathrm{RC}$ & N & 15 & $\mathrm{~K}$ & 200 & $<.05$ & .140 & 30 & -- & .3 & $N$ & -- & 5.0 \\
\hline IV $371 \mathrm{RF}$ & N & 10 & $\mathrm{~N}$ & 200 & $<.05$ & .080 & 5 & -- & .1 & 1 & -- & $\mathrm{N}$ \\
\hline IV $371 \mathrm{RF}$ & N & 15 & $\mathrm{~N}$ & 300 & $<.05$ & .080 & 5 & -- & .1 & $\mathrm{~N}$ & -- & $N$ \\
\hline IV $372 \mathrm{R}$ & N & 50 & 200 & 100 & $<.05$ & .140 & 160 & -- & .3 & $\mathrm{~N}$ & -- & $<5.0$ \\
\hline IV $375 \mathrm{R}$ & N & 20 & $N$ & 50 & $\mathrm{~N}$ & .040 & 10 & -- & $\mathrm{N}$ & N & -- & N \\
\hline IV $375 \mathrm{RA}$ & N & 30 & $\mathrm{~N}$ & 100 & N & .240 & 50 & -- & $\mathrm{N}$ & N & -- & $\mathrm{N}$ \\
\hline IV $375 \mathrm{RB}$ & N & 50 & $\mathrm{~N}$ & 100 & N & .080 & 1,200 & -- & 1.9 & 2 & - & 20.0 \\
\hline IV $375 \mathrm{~K}=$ & N & 70 & $\mathrm{~N}$ & 100 & N & .040 & 90 & -- & $\mathrm{N}$ & $\mathrm{N}$ & - & 5.0 \\
\hline IV $375 R D$ & N & 20 & $\mathrm{~N}$ & 30 & $\mathrm{~N}$ & .080 & 15 & -- & N & $\mathrm{N}$ & -- & 10.3 \\
\hline IV $375 \mathrm{RE}$ & N & 70 & $\mathrm{~N}$ & 200 & $\mathrm{~N}$ & .020 & 80 & -- & N & $\mathrm{N}$ & -- & 5.0 \\
\hline IV $375 \mathrm{RF}$ & $n$ & 10 & $\mathrm{~N}$ & 70 & N & .260 & 5 & -- & N & 5 & -- & 100.0 \\
\hline IV $375 \mathrm{RG}$ & $\mathrm{N}$ & $5 c$ & $N$ & 300 & $\mathrm{~N}$ & .040 & 50 & -- & $\mathrm{N}$ & $\mathrm{N}$ & - & 5.0 \\
\hline IV $375 \mathrm{RH}$ & i & 70 & $\mathrm{~N}$ & 200 & $\mathrm{~N}$ & .020 & 10 & -- & N & $N$ & -- & $\mathrm{N}$ \\
\hline IV 375 R.I & N & 70 & $<200$ & 300 & $\mathrm{~N}$ & .320 & 60 & -- & N & $\mathrm{N}$ & -- & 10.2 \\
\hline IV $375 \mathrm{RJ}$ & N & 15 & 700 & 20 & $<.05$ & .280 & 1,400 & -- & 39.0 & 40 & -- & 60.0 \\
\hline IV $375 R K$ & N & 30 & $\mathbf{N}$ & 150 & $N$ & .040 & $5 C$ & - & .2 & 2 & - & 15.0 \\
\hline IV $375 \mathrm{PL}$ & N & 10 & $\mathrm{~N}$ & $\mathrm{~N}$ & N & $>10.000$ & 50 & -- & 16.0 & 130 & -- & $\mathrm{N}$ \\
\hline IV $375 \mathrm{R}$ : & N & 50 & $N$ & 100 & N & .040 & 15 & -- & .1 & 2 & -- & 5.0 \\
\hline IV $375 \mathrm{RN}$ & $\mathrm{N}$ & 70 & $\mathrm{~N}$ & 70 & $\mathrm{~N}$ & .840 & 30 & - & .2 & 1 & -- & 20.0 \\
\hline IV375RJ & N & 20 & $\mathrm{~N}$ & 100 & N & .300 & 45 & -- & .2 & 1 & -- & 30.0 \\
\hline IV $376 \mathrm{R}$ & N & 70 & $\mathrm{~N}$ & 300 & N & .040 & 100 & -- & .7 & $\mathrm{~N}$ & -- & 5.0 \\
\hline IV $376 \mathrm{RA}$ & $\mathrm{N}$ & 30 & N & 150 & N & $>10.000$ & 10 & -- & $\mathrm{N}$ & 7 & - & 150.0 \\
\hline IV $375 \mathrm{RB}$ & N & 20 & $<200$ & 100 & N & $>10.000$ & 190 & -- & .6 & 25 & -- & 15.0 \\
\hline IV $376 \mathrm{RC}$ & $\mathrm{N}$ & 10 & $N$ & N & N & .060 & 5 & -- & $\mathrm{N}$ & 1 & -- & N \\
\hline $\begin{array}{l}\text { IV } 376 \mathrm{PD} \\
\text { IV } 376 \mathrm{RF}\end{array}$ & $\mathrm{N}$ & 100 & $<200$ & 200 & $\mathrm{~N}$ & $>10.000$ & 280 & -- & .5 & 3 & -- & 55.0 \\
\hline $1 \sqrt{3768 E}$ & $\mathrm{~N}$ & 20 & $\mathrm{~N}$ & 70 & $\mathrm{~N}$ & $<.020$ & 10 & -- & .2 & 5 & -- & 40.0 \\
\hline
\end{tabular}


Table 4 -- Analyses of rock samples collected from the Crater mercury-sulfur-gypsum mineralized area, Inyo county,

Sample

\section{IV $376 \mathrm{RF}$}

IV $376 \mathrm{RG}$

IV $376 \mathrm{RH}$

IV $376 \mathrm{RI}$

I $376 \mathrm{RJ}$

IV $376 \mathrm{RK}$

IV $376 \mathrm{RL}$

IV $376 \mathrm{RH}$

IV $377 \mathrm{R}$

IV $377 \mathrm{FA}$

I $\vee 377 \mathrm{kB}$

IV $377 \mathrm{BC}$

IV $377 \mathrm{RD}$

IV $377 \mathrm{RE}$

IV $377 \mathrm{RF}$

IV $377 R G$

IV $389 \mathrm{P}$

IV $389 \mathrm{RA}$

IV $389 R P$

IV $400 R A$

I V $400 R B$

IV $400 R E$

IV 4 OORD

IV $400 R R$

SV317R 1

SV $317 R 2$

SV $321 R 1$

SV $321 R 2$

SV $322 R 1$

SV $322 R 2$

$\mathrm{SV} 322 \mathrm{R} 3$

SV $334 R 1$

SV $334 R 2$

SV $334 R 3$

SV $334 R 4$

SV $334 R 5$

SV $334 R 6$

SV $334 R 7$

\section{Latitude \\ Longitude Fe-pct.}

$37 \quad 12$ $37 \quad 12 \quad 8$

$\begin{array}{lll}37 & 12 & 11\end{array}$

$\begin{array}{lll}37 & 12 & 5\end{array}$

3712

$\begin{array}{lll}37 & 12 & 5\end{array}$

$\begin{array}{lll}37 & 12 & 5\end{array}$

$\begin{array}{lll}37 & 12 & 4\end{array}$

$37 \quad 10 \quad 31$

$\begin{array}{lll}37 & 10 & 32\end{array}$

$\begin{array}{lll}37 & 10 & 39\end{array}$

$\begin{array}{lll}37 & 10 & 39\end{array}$

$\begin{array}{lll}37 & 10 & 39\end{array}$

$\begin{array}{lll}37 & 10 & 42\end{array}$

$37 \quad 10 \quad 42$

$\begin{array}{lll}37 & 10 & 46\end{array}$

$\begin{array}{lll}37 & 12 & 31\end{array}$

$\begin{array}{llll}37 & 12 & 31\end{array}$

$3712 \quad 31$

$\begin{array}{lll}37 & 12 \quad 49\end{array}$

$37 \quad 14 \quad 36$

$37 \quad 14 \quad 36$

$\begin{array}{llll}37 & 14 & 36\end{array}$

$\begin{array}{lll}37 & 12 \quad 49\end{array}$

371113

$\begin{array}{lll}37 & 11 & 13\end{array}$

$\begin{array}{lll}37 & 1357\end{array}$

$\begin{array}{lll}37 & 13 & 59\end{array}$

$\begin{array}{lll}37 & 14 & 35\end{array}$

$\begin{array}{lll}37 & 14 & 41\end{array}$

$37 \quad 14 \quad 4$

$37 \quad 10 \quad 26$

$\begin{array}{lll}37 & 10 & 26\end{array}$

$\begin{array}{lll}37 & 10 & 36\end{array}$

$37 \quad 10 \quad 36$

$37 \quad 10 \quad 36$

$\begin{array}{lll}37 & 10 & 36 \\ 37 & 10 & 36\end{array}$ $s$

1174322

1174258

$117 \quad 42 \quad 42$

$11742 \quad 42$

$\begin{array}{lll}117 & 42 & 42\end{array}$

$\begin{array}{llll}117 & 42 \quad 42\end{array}$

$\begin{array}{llll}117 & 42 & 34\end{array}$

1174131

1174128

1174125

$\begin{array}{llll}117 & 4125\end{array}$

1174125

$11741 \quad 12$

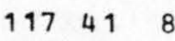

1174159

1174159

1174159

$11741 \quad 15$

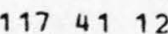

$117 \quad 41 \quad 12$

$117 \quad 4112$

1174915

$117 \quad 4257$

1174257

1174251

1743310

$11743 \quad 5$

$11742 \quad 35$

$117 \quad 42 \quad 35$

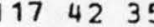

$117 \quad 42 \quad 35$

$117 \quad 42 \quad 35$

$\begin{array}{lll}117 & 42 & 35 \\ 117 & 42 & 35\end{array}$

2.00

15.00

7.00

10.00

5.00

1.50

10.00

.70

.30

.50
2.00

.70

.50

.10
3.00

.70
.07

N

1.50

15.00

.05

7.00

2.00

.30

.05

1.50
.07

20.00

20.00

.07

10.00

1.00
$117 \quad 41 \quad 12$

$$
s
$$

$$
s
$$
$s$

$\mathrm{Mn}-$

$s$

$A g-p p m$

As-ppm

$B-p$

$s$

$\mathrm{Ba}-$

$s$

Be-ppm

5

$\mathrm{Bi}-\mathrm{P}$
$\mathrm{S}$

.50
.07
1.00

10.00

20.00

.005
5.00

5.00

.050

.150

.500
.010

10.00

2.00

20.00

15.00

1.00

1.00

20.00

5.00

.050

$<.002$

.300

.070
.500

7.00

$>20.00$

$>20.00$

$.50>20.00$

10.00

20.00

$>20.00$

10.00

.05

.07

23.00

.50

.10
23.00

.70

.05

20.00

$>20.00$

.70
20.00

$<.05$

$<.02$

$<.05$

.10
.20

.02

5.00

2.00

.20

5.00

$<.05$

.02

.10
$\times .05$

$<.05$

.05

.15

.10

.070

.030

. 015

.100

.030

.030
.050

.050

$>1.000$

.050

.050

.150

.150

.100

.100

.070

.500

.015

.100

300

.500

.02

$<.02$

$<.02$

.05

$<.05$

1. 000

.500
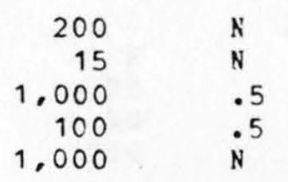

\section{N}

$\mathrm{N}$
20
100
200

5,000

300

700
2.000

$\mathrm{N}$
$\mathrm{N}$
1.0
1.5
5.0

700

2.000

300

300
300

100

200
70

200

200

150

$<10$
10

200

i

700

70

20

10

100
300

500

$2.0 \quad 500$

$2.0 \quad 500$

20

0.000 

Table 4 -- Analyses of rock samples collected from the Crater mercury-sulfur-gypsum mineralized area, Inyo county,

\begin{tabular}{|c|c|c|c|c|c|c|c|c|c|c|c|c|c|}
\hline Sample & $\begin{array}{c}C d-p p m \\
s\end{array}$ & $\begin{array}{c}\mathrm{CO}-\mathrm{ppm} \\
\mathrm{s}\end{array}$ & $\begin{array}{c}\mathrm{Cr}-\mathrm{ppm} \\
\mathrm{s}\end{array}$ & $\begin{array}{c}\mathrm{Cu}-\mathrm{Dpm} \\
\mathrm{s}\end{array}$ & $\begin{array}{c}\mathrm{La}-\mathrm{pDm} \\
\mathrm{s}\end{array}$ & $\begin{array}{c}\mathrm{MO} O \mathrm{ppm} \\
\mathrm{S}\end{array}$ & $\begin{array}{c}N b-p p m \\
s\end{array}$ & $\begin{array}{c}\mathrm{N} 1-\mathrm{pPm} \\
\mathbf{s}\end{array}$ & $\begin{array}{c}\mathrm{Pb}-\mathrm{Ppm} \\
\mathrm{S}\end{array}$ & $\begin{array}{c}S b-p p a \\
s\end{array}$ & $\underset{s}{S c-p p m}$ & $\begin{array}{c}S r-p p m \\
s\end{array}$ & $\begin{array}{c}v-p p m \\
s\end{array}$ \\
\hline IV $376 \mathrm{RF}$ & N & N & $<10$ & $<5$ & N & $\mathrm{N}$ & N & 7 & 200 & N & $\mathbf{N}$ & 200 & 15 \\
\hline IV $376 \mathrm{RG}$ & $\mathrm{N}$ & $\mathrm{N}$ & $<10$ & 7 & $\mathrm{~N}$ & N & N & N & 10 & $\mathrm{~N}$ & $\mathrm{~N}$ & $\mathrm{~N}$ & 10 \\
\hline IV $376 \mathrm{RH}$ & N & $\mathrm{N}$ & 100 & 7 & N & $\mathrm{N}$ & N & 20 & 15 & N & 5 & 500 & 100 \\
\hline IV376RI & $\mathrm{N}$ & 5 & 150 & 50 & 50 & N & $<20$ & 30 & 15 & $N$ & 10 & $\mathrm{~N}$ & 300 \\
\hline IV $376 \mathrm{RJ}$ & $<20$ & 15 & $<10$ & 30 & 50 & 70 & $\mathrm{~N}$ & 200 & 70 & $<100$ & 5 & N & 70 \\
\hline & $\mathrm{N}$ & 5 & 30 & 15 & $<20$ & 15 & N & 150 & 100 & 1,000 & $<5$ & N & 100 \\
\hline IV $376 \mathrm{RL}$ & N & 7 & N & $<5$ & 50 & 7 & $\mathrm{~N}$ & 200 & $\mathrm{~N}$ & 150 & $\mathrm{~N}$ & $\mathrm{~N}$ & 30 \\
\hline IV376R日 & $\mathrm{N}$ & 20 & 100 & 50 & 50 & $\mathrm{~N}$ & $<20$ & 50 & 30 & 200 & 15 & N & 50 \\
\hline IV377F & $N$ & 7 & 20 & $<5$ & $<20$ & $\mathrm{~N}$ & $\mathrm{~N}$ & 5 & 20 & N & $<5$ & 500 & 20 \\
\hline IV $377 \mathrm{RA}$ & N & 20 & 200 & 30 & 150 & $\mathrm{~N}$ & 20 & 50 & 10 & $N$ & 30 & $\mathrm{~N}$ & 100 \\
\hline IV $377 \mathrm{RB}$ & $N$ & $<5$ & 20 & 5 & N & $N$ & N & 5 & 20 & $N$ & $\mathrm{~N}$ & 500 & 15 \\
\hline IV $377 \mathrm{RC}$ & N & 5 & $<10$ & 5 & $\mathrm{~N}$ & $\mathrm{~N}$ & N & 7 & 15 & N & $\mathrm{N}$ & $<100$ & $<10$ \\
\hline IV $377 \mathrm{RD}$ & $\mathrm{N}$ & $<5$ & $<10$ & 5 & $\mathrm{~N}$ & $\mathrm{~N}$ & $N$ & 5 & 30 & N & N & 300 & $<10$ \\
\hline IV $377 \mathrm{RE}$ & $\mathrm{N}$ & 5 & 30 & $<5$ & $<20$ & N & $\mathrm{N}$ & 10 & 15 & N & 5 & 200 & 20 \\
\hline IV377PF & $\mathrm{N}$ & $\mathrm{N}$ & $<10$ & $<5$ & N & $\mathrm{N}$ & N & $<5$ & 20 & $N$ & $\mathrm{~N}$ & 300 & 10 \\
\hline IV $377 \mathrm{RG}$ & N & $\mathrm{N}$ & $<10$ & 5 & N & $\mathrm{N}$ & N & $<5$ & 10 & $\mathbf{N}$ & $<5$ & $\mathrm{~N}$ & 10 \\
\hline IV $389 R$ & N & $\mathrm{N}$ & $<10$ & $<5$ & K & $\mathrm{N}$ & $\mathrm{N}$ & $\mathrm{N}$ & $<10$ & $\mathrm{~N}$ & $\mathrm{~N}$ & $\mathrm{~N}$ & 20 \\
\hline IV $389 \mathrm{RA}$ & N & $\mathrm{s}$ & 20 & 100 & $\mathrm{~N}$ & 15 & $\mathrm{~N}$ & 10 & 15 & 500 & $<5$ & $<100$ & 100 \\
\hline IV389RB & N & $\mathrm{N}$ & $<10$ & 30 & N & $\mathrm{N}$ & $\mathrm{N}$ & 10 & 100 & N & $\kappa$ & $\mathrm{N}$ & 10 \\
\hline IV $400 \mathrm{KA}$ & N & N & 20 & $<5$ & $\mathrm{~N}$ & 15 & 50 & $\mathrm{~N}$ & 20 & N & -- & $N$ & 50 \\
\hline IV $400 R B$ & N & N & $<10$ & $\mathrm{i}$ & 50 & $N$ & N & $\mathrm{N}$ & $\mathrm{N}$ & $N$ & -- & $\mathrm{N}$ & $<10$ \\
\hline IV $400 F C$ & N & 5 & 10 & 10 & $\mathrm{~N}$ & $N$ & $\mathrm{~N}$ & 5 & 50 & $N$ & -- & 100 & 50 \\
\hline IV $400 R D$ & N & 15 & 150 & 500 & 100 & 50 & $\mathrm{~N}$ & 200 & 1,000 & 500 & -- & 1,000 & 700 \\
\hline IV $400 \mathrm{FR}$ & N & $\mathrm{N}$ & $<10$ & $<5$ & $\mathrm{~N}$ & $N$ & N & N & 10 & $\mathrm{~N}$ & $\ldots$ & 100 & 10 \\
\hline SV $317 R 1$ & N & $\mathrm{N}$ & 10 & 10 & N & 20 & $\mathrm{~N}$ & 20 & 10 & 150 & -- & $N$ & 20 \\
\hline SV317R2 & N & $\mathrm{N}$ & 20 & 50 & N & N & N & 100 & 10 & N & - & $N$ & 150 \\
\hline SV321R 1 & $\mathrm{~N}$ & N & 20 & 30 & $\mathrm{~N}$ & 30 & N & 10 & 5,000 & $\mathrm{~N}$ & -- & $\mathrm{N}$ & 20 \\
\hline $\mathrm{SV} 321 \mathrm{R} 2$ & N & N & $<10$ & 5 & $\mathrm{~N}$ & $\mathrm{~N}$ & $\mathrm{~N}$ & $\mathrm{~N}$ & 50 & $y$ & -- & $N$ & 20 \\
\hline$S V 322 R 1$ & $\mathrm{~N}$ & 30 & 300 & 50 & 20 & 10 & $\mathrm{~N}$ & 200 & 50 & N & -- & $\mathrm{N}$ & 70 \\
\hline $\mathrm{SV} 322 \mathrm{R} 2$ & $\mathrm{~N}$ & $\mathrm{~N}$ & 100 & 20 & N & 200 & $\mathrm{~N}$ & 50 & 20 & $\mathrm{~N}$ & -- & $N$ & 2,000 \\
\hline $\mathrm{SV} 322 \mathrm{R} 3$ & $\mathrm{~N}$ & N & $\mathrm{N}$ & $\mathrm{N}$ & N & $\mathrm{N}$ & N & $\mathrm{N}$ & N & $\mathrm{N}$ & -- & $N$ & 20 \\
\hline SV334R 1 & N & 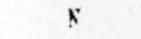 & 50 & 50 & $N$ & $\mathrm{~N}$ & $\mathrm{~N}$ & 50 & N & y & -- & $\mathrm{N}$ & 100 \\
\hline SV334R2 & $\mathrm{N}$ & N & 20 & $<5$ & 150 & 7 & 20 & $\mathrm{~N}$ & 100 & $\mathrm{~N}$ & -- & $\mathrm{N}$ & 52 \\
\hline$S V 334 R 3$ & $N$ & $\mathrm{~N}$ & 2,000 & 20 & 200 & 200 & $\mathrm{~N}$ & 20 & 150 & $\mathrm{~N}$ & -- & 1,500 & 2,000 \\
\hline SV $334 R 4$ & N & $\mathrm{N}$ & 200 & 20 & 150 & 20 & $\mathrm{~N}$ & 50 & $\mathrm{~N}$ & v & - & 100 & 300 \\
\hline SV334R5 & $B$ & $\mathrm{~N}$ & $<10$ & 5 & 150 & 20 & 20 & $\mathrm{~N}$ & 70 & $N$ & -- & $\mathrm{N}$ & 50 \\
\hline SV334R6 & N & $\mathrm{N}$ & 300 & 70 & 150 & 100 & $N$ & 30 & 300 & $\mathrm{~N}$ & -- & 700 & 1.000 \\
\hline SV334R7 & $N^{\prime}$ & $\mathrm{N}$ & 70 & 10 & 100 & 70 & $<20$ & 10 & 10 & $\mathrm{~s}$ & -- & $\mathrm{N}$ & 20 \\
\hline
\end{tabular}


$\begin{aligned} \text { Table } 4 \text {-- Analyses of rock samples collected from the Crater mercury-sulfur-gypsum mineralized area, Inyo County, } & \text { Calfornia--Continued }\end{aligned}$

\begin{tabular}{|c|c|c|c|c|c|c|c|c|c|c|c|c|}
\hline Sample & $\begin{array}{c}H-p p m \\
s\end{array}$ & $\begin{array}{c}Y-p p m \\
s\end{array}$ & $\begin{array}{c}\mathrm{Zn-ppm} \\
\mathrm{s}\end{array}$ & $\begin{array}{c}\mathrm{ZI}-\mathrm{ppm} \\
\mathrm{s}\end{array}$ & $\begin{array}{c}A u-p p m \\
a a\end{array}$ & $\begin{array}{c}\mathrm{Hg}-\mathrm{ppm} \\
\text { inst }\end{array}$ & $\begin{array}{c}2 n-p p m \\
a a\end{array}$ & $\begin{array}{c}A g-p p m \\
a a\end{array}$ & $\begin{array}{c}C d-p p m \\
a a\end{array}$ & $\begin{array}{c}S b-p p d \\
a a\end{array}$ & $\begin{array}{c}A S-P P \mathbb{D} \\
C \mathbb{M}\end{array}$ & $\begin{array}{c}A S-p p \mathbb{P} \\
a a\end{array}$ \\
\hline IV $376 \mathrm{RF}$ & $\mathrm{N}$ & 15 & $\mathbf{N}$ & $<10$ & $\mathrm{~N}$ & .040 & 50 & -- & .2 & 6 & -- & 5.0 \\
\hline IV376RJ & $\mathrm{N}$ & $<10$ & $\mathrm{~N}$ & 300 & $\mathrm{~N}$ & $>10.000$ & $\mathrm{~N}$ & -- & $\mathrm{N}$ & N & -- & 5.0 \\
\hline IV $376 \mathrm{BH}$ & $\mathrm{N}$ & 30 & $<200$ & 70 & $\mathrm{~N}$ & .080 & 80 & -- & .3 & 2 & -- & 5.0 \\
\hline IV $376 \mathrm{RI}$ & $\mathrm{N}$ & 30 & $<200$ & 200 & $\mathrm{~N}$ & $>10.000$ & 100 & -- & .1 & 12 & -- & 160.0 \\
\hline IV $376 \mathrm{RJ}$ & $\mathrm{N}$ & 70 & 2.000 & N & $\mathrm{N}$ & $>10.000$ & 7.600 & -- & 25.0 & 200 & -- & 2.100 .0 \\
\hline & 50 & 30 & 500 & 100 & $\mathrm{~N}$ & $>10.000$ & 650 & -- & .9 & 700 & -- & 880.0 \\
\hline $\begin{array}{l}\text { IV } 376 \mathrm{KL} \\
\mathrm{IV} 376 \mathrm{RM}\end{array}$ & $\mathbf{N}$ & 15 & 2.000 & $\mathrm{~N}$ & .15 & $>10.000$ & 5,500 & -- & .8 & 230 & -- & $2,100.0$ \\
\hline IV $376 \mathrm{RH}$ & $\mathrm{N}$ & 50 & $<200$ & 500 & $\mathrm{~N}$ & $>10.000$ & 85 & -- & .1 & 70 & -- & 20.0 \\
\hline IV $377 R$ & $\mathrm{~N}$ & 20 & $\mathrm{~N}$ & 10 & $\mathrm{~N}$ & .020 & 20 & -- & N & 1 & - & 13.0 \\
\hline IV $377 \mathrm{RA}$ & $\mathrm{N}$ & 70 & $\mathrm{~N}$ & 100 & N & .020 & 40 & -- & N & $\mathrm{N}$ & -- & 5.0 \\
\hline IV $377 \mathrm{FE}$ & $\mathrm{N}$ & 10 & $\mathrm{~N}$ & $<10$ & N & $<.020$ & 20 & -- & N & 1 & -- & N \\
\hline IV $377 \mathrm{RC}$ & $N$ & 15 & $\mathrm{~N}$ & $<10$ & $\mathrm{~N}$ & $<.020$ & 20 & -- & $\mathrm{N}$ & $\mathrm{N}$ & -- & 5.0 \\
\hline IV $377 R D$ & $\mathrm{~N}$ & $<10$ & $\mathrm{~N}$ & N & N & $<.020$ & 5 & -- & N & N & -- & 5.0 \\
\hline IV $377 \mathrm{RE}$ & $\mathrm{N}$ & 15 & $\mathrm{~N}$ & 15 & $\mathrm{~N}$ & $<.020$ & 15 & -- & N & N & -- & $N$ \\
\hline IV377RF & $\mathrm{N}$ & $<10$ & $\mathrm{~N}$ & $\mathrm{~N}$ & $\mathrm{~N}$ & .020 & 60 & -- & .2 & N & -- & 10.0 \\
\hline IV $377 \mathrm{RG}$ & $\mathbf{N}$ & $<10$ & $\mathrm{~N}$ & $<10$ & $N$ & $<.020$ & 10 & -- & N & $N$ & -- & 10.0 \\
\hline IV $389 \mathrm{~K}$ & $\mathrm{~N}$ & 10 & $\mathrm{~N}$ & 200 & $\mathrm{~N}$ & $>10.000$ & 5 & -- & $\mathrm{N}$ & 15 & -- & 15.3 \\
\hline IV $389 \mathrm{RA}$ & $\mathrm{N}$ & 10 & $\mathrm{~N}$ & 70 & .05 & $>10.000$ & 25 & -- & .2 & 320 & -- & $43 C .0$ \\
\hline IV $389 R B$ & $\mathrm{~N}$ & 10 & $<200$ & $\mathrm{~N}$ & $\mathrm{~N}$ & $>10.000$ & 230 & -- & 1.2 & 5 & -- & 130.0 \\
\hline IV $400 \mathrm{KA}$ & N & 10 & N & -- & $N$ & .240 & 5 & -- & . 1 & 5 & -- & N \\
\hline IV $400 \mathrm{RE}$ & $\mathrm{N}$ & $\mathrm{N}$ & $\mathrm{N}$ & -- & N & $>10.000$ & $<5$ & -- & N & $\mathrm{N}$ & -- & . \\
\hline IV $4 \supset 0 \mathrm{PC}$ & N & 15 & $\mathrm{~N}$ & -- & $\mathrm{N}$ & $>10.000$ & 20 & -- & $\mathrm{N}$ & 11 & -- & 25.0 \\
\hline IV $400 R D$ & $\mathrm{~N}$ & 50 & 1,000 & -- & $N$ & $>10.000$ & 1,200 & -- & 16.0 & 350 & -- & 2.300 .0 \\
\hline IV $400 R R$ & $\mathrm{~N}$ & N & $\mathrm{N}$ & -- & $N$ & .660 & 5 & -- & $N$ & $\mathrm{~N}$ & -- & N \\
\hline SV317R 1 & N & N & $\mathrm{N}$ & $\mathrm{N}$ & .05 & 600.000 & 50 & -- & .4 & 180 & -- & 500.0 \\
\hline SV317R2 & $\mathrm{N}$ & 10 & $\mathrm{~N}$ & 50 & $\mathrm{~N}$ & 35.000 & 135 & -- & $>100.0$ & 17 & -- & 300.5 \\
\hline SV $321 \mathrm{R} 1$ & $N$ & 10 & $\mathrm{~N}$ & 500 & $\mathrm{~N}$ & .960 & 10 & -- & $\mathrm{N}$ & 8 & -- & 45.0 \\
\hline SV $321 R 2$ & $\mathrm{~N}$ & 10 & $\mathrm{~N}$ & 150 & $\mathrm{~N}$ & .160 & 30 & -- & N & 10 & -- & 35.0 \\
\hline SV322R 1 & $\mathrm{~N}$ & 30 & $<200$ & 100 & -- & - & 250 & -- & .3 & $\mathrm{~N}$ & -- & 10.1 \\
\hline $\mathrm{SV} 322 \mathrm{R} 2$ & $\mathrm{~N}$ & 15 & $\mathrm{~N}$ & 50 & $\mathrm{~N}$ & .076 & 15 & -- & .2 & 6 & - & 250.0 \\
\hline SV $322 R 3$ & N & N & $\mathrm{N}$ & 50 & N & .040 & $<5$ & -- & N & $\mathrm{N}$ & -- & $N$ \\
\hline SV334R1 & N & $N$ & $\mathrm{~N}$ & 20 & $\mathrm{~N}$ & .500 & 10 & -- & .1 & 6 & -- & 30.0 \\
\hline SV $334 \mathrm{R} 2$ & $\mathrm{~N}$ & 100 & $\mathrm{~N}$ & 1,000 & N & 7.000 & 5 & -- & .2 & 22 & -- & 15.0 \\
\hline SV $334 R 3$ & $\mathrm{~N}$ & 20 & $\mathrm{~N}$ & 200 & N & 16.000 & 40 & -- & .8 & 170 & -- & 1.950 .0 \\
\hline SV $334 R 4$ & N & 70 & $\mathrm{~N}$ & 1.000 & N & 2.000 & 110 & -- & 1.2 & 22 & -- & 200.0 \\
\hline SV $334 R 5$ & $\mathrm{~N}$ & 50 & $\mathrm{~N}$ & 700 & $N$ & 6.000 & $<5$ & -- & N & 36 & -- & 5.0 \\
\hline SV $334 R 6$ & N & $\mathrm{N}$ & $N$ & 15 & N & 2.700 & 50 & -- & .5 & 60 & -- & 750.0 \\
\hline $5 V 334 R 7$ & N & 50 & $N$ & 500 & N & .900 & $<5$ & -- & .1 & 6 & -- & 5.0 \\
\hline
\end{tabular}

